University of Wollongong

Research Online

Faculty of Informatics - Papers (Archive)

Faculty of Engineering and Information

Sciences

2-7-2007

\title{
Quasi-Monte Carlo for Highly Structured Generalised Response Models
}

\author{
I. H. Sloan \\ University of New South Wales \\ F. Y. Kuo \\ University of New South Wales \\ W. T. Dunsmuir \\ University of New South Wales \\ M. Wand \\ University of Wollongong, mwand@uow.edu.au \\ R. S. Womersley \\ University of New South Wales
}

Follow this and additional works at: https://ro.uow.edu.au/infopapers

Part of the Physical Sciences and Mathematics Commons

\section{Recommended Citation}

Sloan, I. H.; Kuo, F. Y.; Dunsmuir, W. T.; Wand, M.; and Womersley, R. S.: Quasi-Monte Carlo for Highly Structured Generalised Response Models 2007.

https://ro.uow.edu.au/infopapers/529

Research Online is the open access institutional repository for the University of Wollongong. For further information contact the UOW Library: research-pubs@uow.edu.au 


\title{
Quasi-Monte Carlo for Highly Structured Generalised Response Models
}

\author{
Abstract \\ Highly structured generalised response models, such as generalised linear mixed models and generalised \\ linear models for time series regression, have become an indispensable vehicle for data analysis and \\ inference in many areas of application. However, their use in practice is hindered by high-dimensional \\ intractable integrals. Quasi-Monte Carlo (QMC) is a dynamic research area in the general problem of high- \\ dimensional numerical integration, although its potential for statistical applications is yet to be fully \\ explored. We survey recent research in QMC, particularly lattice rules, and report on its application to \\ highly structured generalised response models. New challenges for QMC are identified and new \\ methodologies are developed. QMC methods are seen to provide significant improvements compared \\ with ordinary Monte Carlo methods.

\section{Keywords} \\ Generalised Linear Mixed Models, High-dimensional Integration, Lattice Rules, Longitudinal Data Analysis, \\ Maximum Likelihood, Quasi-Monte Carlo, Semiparametric \\ Disciplines \\ Physical Sciences and Mathematics \\ Publication Details \\ This article was originally published as Quasi-Monte Carlo for Highly Structured Generalised Response \\ Models, (in press), 2007.
}




\title{
Quasi-Monte Carlo for Highly Structured Generalised Response Models
}

\author{
BY F.Y. KUO, W.T.M. DUNSMUIR, I.H. SLOAN, M.P. WAND \\ AND R.S. WOMERSLEY \\ School of Mathematics and Statistics, University of New South Wales, \\ Sydney 2052, Australia
}

2 November 2006

\begin{abstract}
Highly structured generalised response models, such as generalised linear mixed models and generalised linear models for time series regression, have become an indispensable vehicle for data analysis and inference in many areas of application. However, their use in practice is hindered by high-dimensional intractable integrals. Quasi-Monte Carlo (QMC) is a dynamic research area in the general problem of high-dimensional numerical integration, although its potential for statistical applications is yet to be fully explored. We survey recent research in QMC, particularly lattice rules, and report on its application to highly structured generalised response models. New challenges for QMC are identified and new methodologies are developed. QMC methods are seen to provide significant improvements compared with ordinary Monte Carlo methods.

Some keywords: Generalised Linear Mixed Models; High-dimensional Integration; Lattice Rules; Longitudinal Data Analysis; Maximum Likelihood; Quasi-Monte Carlo; Semiparametric Regression; Serial Dependence; Time Series Regression.
\end{abstract}

\section{Introduction}

Messy data sets with a generalised response variable abound in many contemporary areas of application. By "generalised response" we mean a response variable that is far from normally distributed, such as a binary, count or heavily skewed variable. This departure from normality is the motivation for the development of generalised linear models and extensions. An example of such data is given in Figure 1 and involves longitudinal measurements on 275 Indonesian children from Diggle, Liang \& Zeger (1995). The response variable is an indicator of respiratory infection. Of interest are the effects of covariates such as vitamin A nourishment. However, regression-type analyses needs to account for correlation among repeated measures on the same child as well a possibly non-linear age effect. 


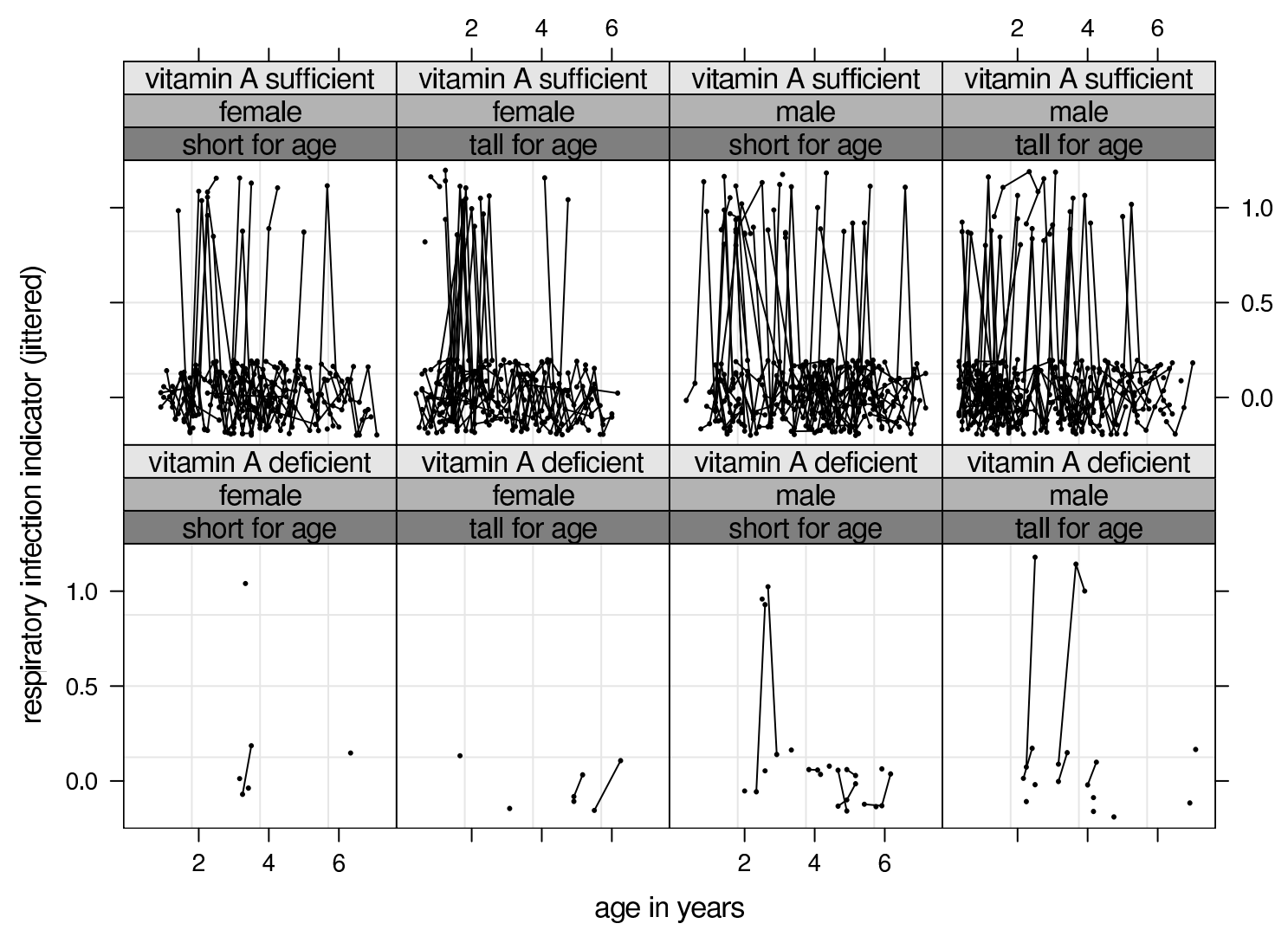

Figure 1: Respiratory infection indicator ( $0=a b s e n t, 1=$ present) versus age in years for a cohort of Indonesian children. Repeated measures on same child are connected by lines. Each panel corresponds to a different combination of three covariates: vitamin A nourishment, gender and height for age. The respiratory infection indicator has been jittered to enhance visualisation.

A useful vehicle for analysis of data such as these is the set of generalised linear mixed models. A random intercept can take care of the within subject correlation. Provided the design matrices are permitted to be of general form then penalised spline basis functions can take care of non-linear covariate effects (e.g. Zhao, Staudenmayer, Coull \& Wand, 2006). However, the likelihood involves intractable integrals of dimension as high as the number of basis functions, usually in the range 10-35. Model fitting and inference requires strategies for dealing with such integrals. The most common approaches involve Laplace approximation (e.g. Breslow \& Clayton, 1993) and Markov Chain Monte Carlo (e.g. Clayton, 1996; Zhao et al., 2006).

In time series regression with generalised response the integration problems are even worse. Davis, Dunsmuir \& Wang (2000) discuss several applications of Poisson regression in time series with serial dependence arising in public health: a series of $T=168$ monthly polio counts and a series of $T=1465$ daily asthma counts. The dimension of the integral, $d=T$, corresponds to the length of the time series - potentially in the hundreds or thousands. This is of the same order as a 360-dimensional integration problem in mathematical finance that was successfully handled by Paskov \& Traub (1995). This phenomenal breakthrough was due to integration technology that has become known as 
quasi-Monte Carlo or QMC.

Like the Monte Carlo method, QMC methods approximate high-dimensional integrals by averages of function values sampled at a number of points. However, instead of generating the sample points randomly, the QMC integration points are chosen deterministically in a clever way to be "better than random": they are designed to achieve a faster rate of convergence, thus are more effective and more efficient in practice.

In this article we investigate the extent to which contemporary QMC can handle highdimensional integrals arising in highly structured generalised response models. A necessary step before applying any QMC algorithm is to transform the integrand into the unit cube. It is seen that this transformation, which relates closely to the technique of importance sampling, plays a crucial role in QMC integration. Extensive numerical studies show that QMC does have something to offer, in comparison to ordinary Monte Carlo integration, for highly structured generalised response models.

Pan \& Thompson $(1998,2004,2006)$ and Al-Eid \& Pan (2005) describe the use of QMC for generalised linear mixed model fitting. They used the "square root sequence" advocated by Fang \& Wang (1994) rather than the recent "lattice" methods studied here. Hickernell, Lemieux \& Owen (2005) contains a recent survey of QMC for a statistical readership.

Section 2 provides a summary of highly structured generalised response models and the integration challenges that the log-likelihood presents. Section 3 surveys QMC from its elements up to its most recent developments. QMC approaches to the aforementioned log-likelihood integrals are described in Section 4. Numerical experiments are documented in Section 5. A discussion of the optimisation procedure is given in Section 6. Section 7 contains a brief summary and Section 8 contains additional technical details.

\section{Highly Structured Generalised Response Models}

Let $\mathbf{y}$ be a generalised response vector for which a model in terms of several predictors is sought. The most common generalised response situations are binary $\mathbf{y}$, which results in a conditional Bernoulli likelihood structure; and y containing counts, in which case conditional Poisson likelihood structure is often assumed. A rich class of such models is

$$
\begin{aligned}
f(\mathbf{y} \mid \mathbf{w})=\quad \exp \left\{\mathbf{y}^{\top}(\mathbf{X} \boldsymbol{\beta}+\mathbf{W} \mathbf{w})-\mathbf{1}^{\top} b(\mathbf{X} \boldsymbol{\beta}+\mathbf{W} \mathbf{w})+\mathbf{1}^{\top} c(\mathbf{y})\right\}, \\
\mathbf{w} \text { i.i.d. } \quad N(\mathbf{0}, \mathbf{\Sigma}) .
\end{aligned}
$$

Setting $b(x)=\log \left(1+e^{x}\right)$ and $c(x)=0$ in (1) gives the Bernoulli conditional density with conditional mean $\mathrm{E}(\mathbf{y} \mid \mathbf{w})=\operatorname{logit}^{-1}(\mathbf{X} \boldsymbol{\beta}+\mathbf{W w})$. The Poisson conditional density with conditional mean $\mathrm{E}(\mathbf{y} \mid \mathbf{w})=\exp (\mathbf{X} \boldsymbol{\beta}+\mathbf{W} \mathbf{w})$ corresponds to $b(x)=e^{x}$ and $c(x)=\log (1 / x !)$. The model parameters are $\boldsymbol{\beta}$ and $\boldsymbol{\Sigma}$ although it is common to impose further parametric structure on $\Sigma$. The matrix $\mathbf{X}$ is a design matrix corresponding to the effects in $\beta$. The matrix $\mathbf{W}$ could be a design matrix or simply the identity matrix of appropriate dimension. Since there is a wide range of choices for $\mathbf{W}$ and $\boldsymbol{\Sigma}$ we call such models highly structured. Special cases include generalised linear mixed models (e.g. McCulloch \& Searle, 2000) and generalised linear models for time series regression (e.g. Davis, Dunsmuir \& Wang, 1999). Sections 2.1 - 2.3 provide specific illustrations. 
Models of the form (1) are typically fitted by using maximum likelihood or hierarchical Bayes methodology. We will focus on the likelihood approach, although the ideas are extendible to Bayesian approaches. The log-likelihood of $(\boldsymbol{\beta}, \boldsymbol{\Sigma})$ is

$$
\begin{gathered}
\ell(\boldsymbol{\beta}, \boldsymbol{\Sigma})=\log \int_{\mathbb{R}^{d}} \exp \left\{\mathbf{y}^{\top}(\mathbf{X} \boldsymbol{\beta}+\mathbf{W} \mathbf{w})-\mathbf{1}^{\top} b(\mathbf{X} \boldsymbol{\beta}+\mathbf{W} \mathbf{w})-\frac{1}{2} \mathbf{w}^{\top} \boldsymbol{\Sigma}^{-1} \mathbf{w}\right\} \mathrm{d} \mathbf{w} \\
-\frac{1}{2} \log |\mathbf{\Sigma}|-\frac{d}{2} \log (2 \pi)+\mathbf{1}^{\top} c(\mathbf{y})
\end{gathered}
$$

where $d$ is the dimension of $\mathbf{w}$. However, its computation is thwarted by the intractable integral comprising the first term. By the end of this section we will have described some situations where the integral factorises into integrals of dimension of about $1-3$; others that require integration over dimensions in the tens; and some that require integration over a space having the same dimension as the sample size. The second and third situations pose an obvious challenge.

A simple, and often useful, approximation to $\ell(\beta, \Sigma)$ arises from the Laplace method. This involves a multivariate normal approximation to the integrand via a second order Taylor series approximation of the exponent about its stationary point (e.g. Breslow \& Clayton, 1993). The resulting approximation is

$$
\begin{aligned}
\ell(\boldsymbol{\beta}, \boldsymbol{\Sigma}) \approx \mathbf{y}^{\top} & \left(\mathbf{X} \boldsymbol{\beta}+\mathbf{W} \mathbf{w}^{*}\right)-\mathbf{1}^{\top} b\left(\mathbf{X} \boldsymbol{\beta}+\mathbf{W w}^{*}\right)-\frac{1}{2} \mathbf{w}^{* \top} \boldsymbol{\Sigma}^{-1} \mathbf{w}^{*} \\
& -\frac{1}{2} \log \left|\mathbf{I}+\boldsymbol{\Sigma} \mathbf{W}^{\top} \operatorname{diag}\left\{b^{\prime \prime}\left(\mathbf{X} \boldsymbol{\beta}+\mathbf{W w}^{*}\right)\right\} \mathbf{W}\right|+\mathbf{1}^{\top} c(\mathbf{y})
\end{aligned}
$$

where $\mathbf{w}^{*}$ is a solution to $\mathbf{w}=\mathbf{\Sigma} \mathbf{W}^{\top}\left\{\mathbf{y}-b^{\prime}(\mathbf{X} \boldsymbol{\beta}+\mathbf{W} \mathbf{w})\right\}$. A commonly used further approximation for estimation of $\boldsymbol{\beta}$ is one which assumes that the determinant term is effectively constant as a function of $\beta$ (Breslow \& Clayton, 1993).

\subsection{Generalised Linear Mixed Models}

Generalised linear mixed models (GLMM) have become very popular in Applied Statistics due to their handling a variety of complications arising in contemporary data analysis and inference. A survey of 20th Century work in the area is provided by McCulloch \& Searle (2000). Recent books demonstrating the breadth of GLMM include Ruppert, Wand \& Carroll (2003) and Skrondal \& Rabe-Hesketh (2004).

An important class of GLMM corresponds to (1) with $\mathbf{w}$ set to $\mathbf{u}$, a random effects vector. The $\mathbf{W}$ matrix would then correspond to the design matrix attached to $\mathbf{u}$, usually denoted by $\mathbf{Z}$. Lastly, $\Sigma$ corresponds to the covariance matrix of the random effects and is often denoted by $\mathbf{G}$. In this section we will use the $\mathbf{Z}$ and $\mathbf{G}$, rather than $\mathbf{W}$ and $\Sigma$, to conform with the GLMM literature. The GLMM version of (1) is then

$$
\begin{aligned}
f(\mathbf{y} \mid \mathbf{u}) & =\exp \left\{\mathbf{y}^{\top}(\mathbf{X} \boldsymbol{\beta}+\mathbf{Z u})-\mathbf{1}^{\top} b(\mathbf{X} \boldsymbol{\beta}+\mathbf{Z u})+\mathbf{1}^{\top} c(\mathbf{y})\right\}, \\
\mathbf{u} \text { i.i.d. } & N(\mathbf{0}, \mathbf{G}) .
\end{aligned}
$$

Distinguishing features between this model and its generalisation (1) are: the length of the random effects vector $\mathbf{u}$ is typically of length much smaller than the response vector $\mathbf{y}$, and the covariance matrix $\mathbf{G}$ has relatively simple structure and does contain forms 
arising in time series modelling, such as Toeplitz structure. For many important GLMMs, $\mathbf{G}$ is simply a diagonal matrix with only a few distinct diagonal entries.

Recently Zhao et al. (2005) examined Markov Chain Monte Carlo fitting of a hierarchical Bayes formulation of (3). They made a case for breaking up the linear predictor into sub-components that handle the various covariance structures used in longitudinal data modelling, smoothing and spatial statistics. Considered were decompositions of the design structure such as

$$
\mathbf{X} \boldsymbol{\beta}+\mathbf{Z u}=\mathbf{X}^{\mathrm{R}} \boldsymbol{\beta}^{\mathrm{R}}+\mathbf{Z}^{\mathrm{R}} \mathbf{u}^{\mathrm{R}}+\mathbf{X}^{\mathrm{G}} \boldsymbol{\beta}^{\mathrm{G}}+\mathbf{Z}^{\mathrm{G}} \mathbf{u}^{\mathrm{G}}=\mathbf{X}^{\mathrm{R}} \boldsymbol{\beta}^{\mathrm{R}}+\mathbf{Z}^{\mathrm{R}} \mathbf{u}^{\mathrm{R}}+\mathbf{X}^{\mathrm{G}} \boldsymbol{\beta}^{\mathrm{G}}+\sum_{\ell=1}^{L} \mathbf{Z}_{\ell}^{\mathrm{G}} \mathbf{u}_{\ell}^{\mathrm{G}}
$$

where

$$
\mathbf{X}^{\mathrm{R}} \equiv\left[\begin{array}{c}
\mathbf{X}_{1}^{\mathrm{R}} \\
\vdots \\
\mathbf{X}_{m}^{\mathrm{R}}
\end{array}\right], \quad \mathbf{Z}^{\mathrm{R}} \equiv \underset{1 \leq i \leq m}{\operatorname{blockdiag}}\left(\mathbf{X}_{i}^{\mathrm{R}}\right), \quad \mathbf{Z}^{\mathrm{G}}=\left[\begin{array}{lll}
\mathbf{Z}_{1}^{\mathrm{G}} & \cdots & \mathbf{Z}_{L}^{\mathrm{G}}
\end{array}\right]
$$

with

$$
\operatorname{Cov}\left(\mathbf{u}^{\mathrm{R}}\right)=\underset{1 \leq i \leq m}{\operatorname{blockdiag}}\left(\Sigma^{\mathrm{R}}\right) \quad \text { and } \quad \operatorname{Cov}\left(\mathbf{u}^{\mathrm{G}}\right)=\underset{1 \leq \ell \leq L}{\operatorname{blockdiag}}\left(\sigma_{u \ell}^{2} \mathbf{I}\right) .
$$

The design matrices and parameter vectors with superscript " $R$ " correspond to random intercepts and slopes, as typically used for repeated measures of data on $m$ groups with sample sizes $n_{1}, \ldots, n_{m}$. For $1 \leq i \leq m, \mathbf{X}_{i}^{\mathrm{R}}$ is a $n_{i} \times d^{\mathrm{R}}$ matrix for the random design corresponding to the $i$ th group, $\Sigma^{\mathrm{R}}$ is an unstructured $d^{\mathrm{R}} \times d^{\mathrm{R}}$ covariance matrix. The design matrices and parameter vectors with superscript " $G$ " correspond to general design matrix structure. This allows, for example, the incorporation of non-linear covariate effects via the mixed model representation of penalised splines (Wand, 2003).

In longitudinal data analysis the $\mathbf{Z}^{\mathrm{G}} \mathbf{u}^{\mathrm{G}}$ component is usually absent and $d^{\mathrm{R}}$ is a small integer, typically in the range 1-3. For example, a single covariate Poisson mixed model with random intercept:

$$
y_{i j} \text { i.i.d. Poisson }\left\{\exp \left(\beta_{0}+\beta_{1} x_{i j}+U_{i}\right)\right\}, \quad U_{i} \text { i.i.d. } N\left(0, \sigma_{U}^{2}\right), \quad 1 \leq j \leq n_{i}, 1 \leq i \leq m,
$$

corresponds to $d^{\mathrm{R}}=1$ and $\mathbf{X}_{i}^{\mathrm{R}}$ equalling the $n_{i} \times 1$ vector of ones. The log-likelihood (2) then reduces to

$$
\begin{aligned}
\ell\left(\beta_{0}, \beta_{1}, \sigma_{U}^{2}\right)= & \sum_{i=1}^{m} \sum_{j=1}^{n_{i}}\left\{y_{i j}\left(\beta_{0}+\beta_{1} x_{i j}\right)-\log \left(y_{i j} !\right)\right\}-\frac{m}{2} \log \left(2 \pi \sigma_{U}^{2}\right) \\
& +\sum_{i=1}^{m} \log \int_{-\infty}^{\infty} \exp \left(\sum_{j=1}^{n_{i}} y_{i j} U_{i}-e^{\beta_{0}+\beta_{1} x_{i j}+U_{i}}-\frac{U_{i}^{2}}{2 \sigma_{U}^{2}}\right) \mathrm{d} U_{i}
\end{aligned}
$$

and the remaining intractable integrals are one-dimensional. However, if (5) is extended to the semiparametric model

$$
y_{i j} \text { i.i.d. Poisson }\left\{\exp \left(\beta_{0}+\beta_{1} x_{i j}+f\left(s_{i j}\right)+U_{i}\right)\right\}
$$


where $f(s)=\beta_{2} s+\sum_{k=1}^{K} u_{k}\left(s-\kappa_{k}\right)_{+}$is a $K$-knot penalised spline model for $f$ with $u_{k}$ i.i.d. $N\left(0, \sigma_{u}^{2}\right)$ then the log-likelihood is

$$
\begin{aligned}
& \ell\left(\beta_{0}, \beta_{1}, \beta_{2}, \sigma_{U}^{2}, \sigma_{u}^{2}\right) \\
& =\sum_{i=1}^{m} \sum_{j=1}^{n_{i}}\left\{y_{i j}\left(\beta_{0}+\beta_{1} x_{i j}+\beta_{2} s_{i j}\right)-\log \left(y_{i j} !\right)\right\}-\frac{m}{2} \log \left(2 \pi \sigma_{U}^{2}\right)-\frac{K}{2} \log \left(2 \pi \sigma_{u}^{2}\right) \\
& +\log \int_{\mathbb{R}^{K}}\left(\prod_{i=1}^{m} \int_{-\infty}^{\infty} \exp \left\{\sum_{j=1}^{n_{i}} y_{i j} U_{i}-e^{\beta_{0}+\beta_{1} x_{i j}+\beta_{2} s_{i j}+U_{i}+\sum_{k=1}^{K} u_{k}\left(s_{i j}-\kappa_{k}\right)_{+}}-\frac{U_{i}^{2}}{2 \sigma_{U}^{2}}\right\} \mathrm{d} U_{i}\right) \\
& \times \exp \left\{\sum_{i=1}^{m} \sum_{j=1}^{n_{i}} \sum_{k=1}^{K} y_{i j} u_{k}\left(s_{i j}-\kappa_{k}\right)_{+}-\sum_{k=1}^{K} \frac{u_{k}^{2}}{2 \sigma_{u}^{2}}\right\} \mathrm{d} u_{1} \cdots \mathrm{d} u_{K},
\end{aligned}
$$

The dominating intractable integral is over $\mathbb{R}^{K}$.

Starting mainly with the Laplace approximation papers: Breslow \& Clayton (1993) and Wolfinger \& O'Connell (1993), there has been a great deal of research and software development for GLMM. Chapter 10 of McCulloch \& Searle (2000) surveys the earlier literature. Zhao et al. (2006) reflects contemporary work in GLMM. The SAS procedure PROC NLMIXED (SAS Institute, Inc., 2006) uses quadrature to evaluate low-dimensional integrals such as those arising in (6). Markov Chain Monte Carlo packages based on the BUGS sampling engine (BUGS Project, 2006) have been successfully used in Bayesian GLMMs involving higher-dimensional integrals; see for example Crainiceanu, Ruppert \& Wand (2005), Gurrin, Scurrah \& Hazelton (2005) and Zhao et al. (2006).

\subsection{Generalised Linear Models for Longitudinal Data with Serial Dependence.}

Models of this type are emerging more frequently in the longitudinal data analysis literature - for example see Diggle, Heagerty, Liang \& Zeger (2002). Here

$$
\mathbf{W}=\mathbf{I}, \quad \mathbf{w}=\mathbf{Z}^{\mathrm{R}} \mathbf{u}^{\mathrm{R}}+\boldsymbol{\alpha}
$$

where $\mathbf{Z}^{\mathrm{R}}$ and $\mathbf{u}^{\mathrm{R}}$ represent the random effects components and $\boldsymbol{\alpha}^{\top}=\left(\boldsymbol{\alpha}_{1}^{\top}, \ldots, \boldsymbol{\alpha}_{m}^{\top}\right)$ where

$$
\boldsymbol{\alpha}_{i} \text { i.i.d. } N\left(\mathbf{0}, \boldsymbol{\Gamma}_{i}\right), \quad i=1, \ldots, m
$$

allow for serial dependence within the time ordered repeated measures on the $m$ cases. The covariance matrix $\boldsymbol{\Gamma}_{i}=\boldsymbol{\Gamma}_{i}(\boldsymbol{\lambda})$ is a $n_{i} \times n_{i}$ Toeplitz corresponding to an assumption of stationary serial dependence and is specified using a finite vector of parameters $\lambda$ which is the same for all values of $i$. Let

$$
\Gamma=\underset{1 \leq i \leq m}{\operatorname{blockdiag}}\left(\boldsymbol{\Gamma}_{i}\right)
$$

Computation of the log-likelihood for this model requires computation of $m$ integrals of the type (above) with maximum dimension $d=\max _{i}\left(n_{i}\right)$. Diggle et al. (2002) discuss computational approaches but do not present examples in which both $\mathbf{Z}^{\mathrm{R}} \mathbf{u}^{\mathrm{R}}$ and $\boldsymbol{\alpha}$ are present in the model simultaneously. 
The class of models just considered may also be appropriate for the situation in which $m$ time series of equal length $n_{i} \equiv n$ are observed. For example in Bernat, Dunsmuir \& Wagenaar (2004) the impact of reduction of legal blood alcohol concentration from 0.1 to 0.08 on single vehicle night time fatalities was investigated in the United States. In that study monthly time series of fatalities in $m=19$ states each of length $n_{i}=72$ were analysed using a Poisson mixed model regression with random effects for before and after the change in legal blood alcohol levels. In some of the states significant autocorrelation existed. Other examples that we have encountered include the analysis of monthly suicide counts over a 30 year period in all of the states. Here $n_{i} \equiv 360$ and $m \approx 50$. These situations differ somewhat from the longitudinal data situation in that $m$ is typically smaller and the $n_{i}$ are typically much larger than in the longitudinal setting.

\subsection{Generalised Linear Models for Time Series Regression}

For this paper we will investigate the calculation of a single integral corresponding to a single series in Section 2.2. These single time series models have been extensively studied, in their own right, in recent years. An understanding of the calculation of those integrals required for computing the likelihood in the single series setting will also be necessary for the multiple time series and longitudinal examples of Section 2.2. They correspond to a single ( $m=1$ in the previous model) time series $Y_{1}, \ldots, Y_{T}$ of observations which, conditional on an observed sequence of vectors of regressors, $\left\{x_{t}\right\}$, and an unobserved stationary latent process $\left\{w_{t}\right\}$, are observations from an exponential family model or closely related family such as negative binomial. Throughout this paper we assume that the latent process is the autoregression of degree $p$, referred to as the $\operatorname{AR}(p)$ model henceforth,

$$
w_{t}=\phi_{1} w_{t-1}+\cdots+\phi_{p} w_{t-p}+\eta_{t}
$$

where $\eta_{t}$ i.i.d. $N\left(0, \sigma^{2}\right)$. Here $\mathbf{w}$ is a $d=T$ dimensional vector with multivariate normal distribution specified to have mean vector $\mathbf{0}$ and covariance matrix $\Sigma=\operatorname{cov}(\mathbf{w})$ with $(s, t)$ th element given by the autocovariance at lag $|s-t|$ for the above $\operatorname{AR}(p)$ process. Let $\mathbf{V}=\Sigma^{-1}$ and denote the parameters specifying the covariances as $\theta=\left(\phi_{1}, \ldots, \phi_{p}, \sigma^{2}\right)$ then the log-likelihood of the observed data is as given above with $\mathbf{W}=\mathbf{I}$. Note that the integral required to be calculated is $d$-dimensional with $d=T$. It is not uncommon to have the length $T$ of the time series in the thousands for realistic applications that arise in public health or financial econometrics.

Various simulation based methods currently available for computing the required integral are reviewed in Davis and Rodriguez-Yam (2005). Techniques reviewed include the various computationally intensive methods of importance sampling, Monte Carlo EM, and Monte Carlo Newton-Raphson. Davis and Rodriguez-Yam (2005) introduce an approximation, based on the second order Taylor series expansion, to the conditional density of $w_{t}$ given the count observations $y_{t}$ and exploit the computationally efficient innovations algorithm to evaluate the resulting approximate likelihood. They also use this approximation to develop an approximate importance sampling technique which is computationally much faster than importance sampling. Application to a stochastic 
volatility model for 946 daily Pound-Dollar exchange rates is presented to illustrate the use of their methods beyond the exponential family setting discussed here.

\section{Elements of Quasi-Monte Carlo Methods}

In this section we provide a brief introduction to quasi-Monte Carlo (QMC) methods, with a focus on recent developments in lattice rules. We start our discussion with the Monte Carlo method, to which the QMC methods are related in a natural way.

\subsection{The Monte Carlo Method and Importance Sampling}

Consider an integral

$$
\int_{\mathbb{R}^{d}} g(\mathbf{w}) p(\mathbf{w}) \mathrm{d} \mathbf{w}
$$

where $\mathbf{w}=\left(w_{1}, \ldots, w_{d}\right)^{\top}$ is a $d$-dimensional vector and $p$ is some multivariate probability distribution function. Integrals of the form (7) often arise from multivariate expected values, and in many cases $p$ is the multivariate normal density

$$
(2 \pi)^{-d / 2}|\boldsymbol{\Sigma}|^{-1 / 2} \exp \left\{-\frac{1}{2}(\mathbf{w}-\boldsymbol{\mu})^{\top} \boldsymbol{\Sigma}^{-1}(\mathbf{w}-\boldsymbol{\mu})\right\}
$$

with mean $\boldsymbol{\mu}$ and covariance matrix $\boldsymbol{\Sigma}$. The integral in the log-likelihood (2) is one example; many finance problems also have integrals of this form. The classical Monte Carlo method approximates (7) by an average of function values of $g$

$$
\frac{1}{N} \sum_{i=1}^{N} g\left(\boldsymbol{\xi}_{i}\right)
$$

where $\xi_{1}, \ldots, \xi_{N} \in \mathbb{R}^{d}$ are $N$ independent random samples drawn from the distribution $p$. The expected error is of order $\mathcal{O}\left(N^{-1 / 2}\right)$ and the efficiency depends on how easy it is to sample from the distribution $p$ and how well $p$ captures the features of the integrand $g(\mathbf{w}) p(\mathbf{w})$. More precisely, the root mean square expected error is $\sigma(g) / \sqrt{N}$, where

$$
\sigma^{2}(g):=\int_{\mathbb{R}^{d}} g^{2}(\mathbf{w}) p(\mathbf{w}) \mathrm{d} \mathbf{w}-\left(\int_{\mathbb{R}^{d}} g(\mathbf{w}) p(\mathbf{w}) \mathrm{d} \mathbf{w}\right)^{2},
$$

which we shall refer to as the variance of $g$.

For a more general integral

$$
\int_{\mathbb{R}^{d}} f(\mathbf{w}) \mathrm{d} \mathbf{w}
$$

the integrand $f$ may not have the obvious form of some function times a distribution, or perhaps it has such a form but the distribution does not reflect the features of $f$. In these situations it is up to the user to choose an appropriate distribution $p$ and rewrite (9) in the form of (7), with $g(\mathbf{w})=f(\mathbf{w}) / p(\mathbf{w})$. If an attempt is made to minimise $\sigma(g)$ then this technique is known as importance sampling and $p$ is referred to as the importance sampling distribution. In practice, it is convenient if the sampling distribution $p$ is a product of univariate probability distribution functions. For example, if $p$ is a normal distribution (8), then a change of variable with $\mathbf{z}=\mathbf{A}^{-1}(\mathbf{w}-\boldsymbol{\mu})$ will do the trick, where $\mathbf{A}$ is 
a factorisation of $\boldsymbol{\Sigma}=\mathbf{A} \mathbf{A}^{\top}$. We shall assume for the remainder of this subsection that $p(\mathbf{w}):=\prod_{j=1}^{d} \psi\left(w_{j}\right)$, where $\psi$ is a univariate probability distribution.

Importance sampling can be thought of as a change of variables. Let $\Psi: \mathbb{R} \rightarrow[0,1]$ denote the cumulative distribution function of $\psi$, that is, $\Psi(w)=\int_{-\infty}^{w} \psi(t) \mathrm{d} t$, and let $\Psi^{-1}$ denote its inverse. Using the substitution $\mathbf{z}=\Psi(\mathbf{w}):=\left(\Psi\left(w_{1}\right), \ldots, \Psi\left(w_{d}\right)\right)^{\top}$, the integral (7) can be transformed into an integral over the unit cube

$$
\int_{[0,1]^{d}} g\left(\Psi^{-1}(\mathbf{z})\right) \mathrm{d} \mathbf{z},
$$

where $\Psi^{-1}(\mathbf{z}):=\left(\Psi^{-1}\left(z_{1}\right), \ldots, \Psi^{-1}\left(z_{d}\right)\right)^{\top}$. The Monte Carlo method in effect approximates this integral by

$$
\frac{1}{N} \sum_{i=1}^{N} g\left(\Psi^{-1}\left(\boldsymbol{\xi}_{i}\right)\right),
$$

where now $\xi_{1}, \ldots, \xi_{N} \in[0,1]^{d}$ are i.i.d. uniform random samples drawn from $[0,1]^{d}$. In other words, the points are first drawn from a uniform distribution in $[0,1]^{d}$ and then mapped into $\mathbb{R}^{d}$ using $\Psi^{-1}$. This is equivalent to the earlier discussion, where the points are generated directly from the distribution $p$. For the present approach to work in practice, we must have a way to evaluate $\Psi^{-1}$. Thus this approach is rarely used explicitly by people following the Monte Carlo strategy.

Now we come to the point where quasi-Monte Carlo methods depart from the Monte Carlo method. QMC methods are designed to improve upon the effectiveness and efficiency of the Monte Carlo method. They take the same form as the Monte Carlo method in the unit cube $[0,1]^{d}$, but instead of sampling the points randomly from a uniform distribution, the points are chosen deterministically in a clever way so that a rate of convergence of order $\mathcal{O}\left(N^{-1}(\log N)^{d}\right)$ or better is achieved. Since all QMC methods are defined in the unit cube, to apply QMC methods to a particular integral, one must first transform the integral into the unit cube. From our earlier discussion, it should be clear that importance sampling, with an appropriately chosen distribution, will give us the transformation we need. For the QMC strategy to succeed it is crucial that we can evaluate $\Psi^{-1}$, either analytically or numerically.

\subsection{Low-discrepancy Point Sets}

Assume now that original integral in $\mathbb{R}^{d}$ has been transformed into an integral over the unit cube $[0,1]^{d}$. We consider integrals of the form

$$
\text { If }:=\int_{[0,1]^{d}} f(\mathbf{z}) \mathrm{d} \mathbf{z} .
$$

Then QMC methods approximate such integrals by

$$
Q_{N} f:=\frac{1}{N} \sum_{i=1}^{N} f\left(\boldsymbol{\xi}_{i}\right),
$$


where $\xi_{1}, \ldots, \xi_{N}$ are points from the unit cube $[0,1]^{d}$ chosen deterministically to be "more uniform than random". While the root mean square MC error is $\sigma(f) / \sqrt{N}$ where $\sigma^{2}(f)=$ $I f^{2}-(I f)^{2}$, QMC error bounds typically take the form

$$
\left|I f-Q_{N} f\right| \leq D\left(\xi_{1}, \ldots, \xi_{N}\right) V(f)
$$

where $D\left(\xi_{1}, \ldots, \xi_{N}\right)$ is some discrepancy, which measures the quality (the "uniformity") of the points, and $V(f)$ is a measure of the variation of $f$. For example, the classical Koksma-Hlawka inequality has QMC error bounded by the so-called star discrepancy of the point set and the variation of $f$ in the sense of Hardy and Krause (e.g. Niederreiter, 1992). Error bounds of this form separate the dependence on the point sets from the dependence on the integrand. In general we do not have control over the integrand, and so we choose QMC points that make the discrepancy as small as possible. This is the principle behind all constructions of QMC methods.

One may ask the question: if the aim is to achieve high uniformity, why not just divide the unit interval in each coordinate direction into $n-1$ equal segments and take the $n^{d}$ grid points as our integration points? Clearly this is infeasible even for $n=2$ if $d$ is say 100. Another answer is that the projection of these $N=n^{d}$ points collapse down to just $n$ distinct values in each coordinate direction, and thus for a function that depends only on a single component of $\mathbf{z}$ we receive only the benefit of having $n$ points, yet have $n^{d}$ costly function evaluations. More generally, one can apply a one-dimensional integration rule such as the Simpson rule in each coordinate direction to form what is called a product rule. However, even if the chosen one-dimensional rule has error of order $\mathcal{O}\left(n^{-r}\right)$, the error expressed in terms of $N$ is only of order $\mathcal{O}\left(N^{-r / d}\right)$. In other words, the cost (in terms of function evaluations) for a given level of accuracy increases exponentially in $d$. It is this curse of dimensionality that makes product rules useless for high dimensional integrals.

QMC point sets with discrepancy of order $\mathcal{O}\left(N^{-1}(\log N)^{d}\right)$ or better are collectively known as low-discrepancy point sets. This includes, for example, the well known Sobol' sequences and Niederreiter sequences. These are "sequences" because additional points can be added at any time. Their "extensibility" in $N$ makes them very attractive in practice: if at any stage we decide to increase the number of points in our QMC approximation, we only need to evaluate the function at the additional points. However, point sets which are not extensible in $N$ often have smaller discrepancies.

Digital nets and lattice rules are the two foci in recent QMC research. They represent two different strategies for achieving high uniformly of the points in the unit cube. For a survey of earlier works, see Niederreiter (1992) and Sloan \& Joe (1994). Note that the definition of digital nets and lattice rules do not explicitly forbid product grids. Instead product grids are excluded by the design concepts based on minimising various forms of discrepancy measures.

The concept behind digital nets is all about having the right number of points in various sub-divisions of the unit cube. Figure 2(a) shows the first 64 points of a 2-dimensional Sobol' sequence, which is an example of a digital $(0,6,2)$-net in base 2 . If we divide the 
unit square into 64 strips with width $1 / 64$ in either direction then each strip contains exactly one point (with points on the boundary counting towards the next strip). Similarly if we divide the unit square into 64 squares, we get exactly one point in each square. In fact as long as the unit square is divided into 64 rectangles of the same shape and size, each rectangle will include exactly one point. The quality of a " $(t, m, s)$-net in base $b^{\prime \prime}$ rests upon its $t$-value: the smaller $t$ is, the finer the sub-divisions can be, while preserving the uniformity described above. (The ideal case above corresponds to $t=0$. In a general $(t, m, s)$-net in base $b$ there are $b^{m}$ points in total, with $b^{t}$ points in each sub-division. The dimension is typically denoted by $s$ rather than $d$.) See Niederreiter (2005) for a survey of recent works on the construction of $(t, m, s)$-net.

Lattice rules have a different kind of uniformity: the points of a lattice rule form a group under the operation of addition modulo the integers. One way to visualise a lattice point set is to think of a sheared product grid where the axes have been stretched and rotated under certain constraints. The oldest and simplest kind of lattice rules, now known as rank-1 lattice rules, are uniquely specified by the choice of a generating vector $\boldsymbol{\eta}=\left(\eta_{1}, \ldots, \eta_{d}\right)$, which is an integer vector having no factor in common with $N$. More precisely, the lattice points are given by $\xi_{i}=\operatorname{frac}(i \boldsymbol{\eta} / N)$ for $i=1, \ldots, N$, where $\operatorname{frac}(\mathbf{x})$ is the vector obtained by replacing each component of the vector $\mathrm{x}$ by its fractional parts. Figure 2(b) shows a "Fibonacci" lattice rule with $N=55$ and $\boldsymbol{\eta}=(1,34)$.

Figure 2: Digital net versus lattice rule.

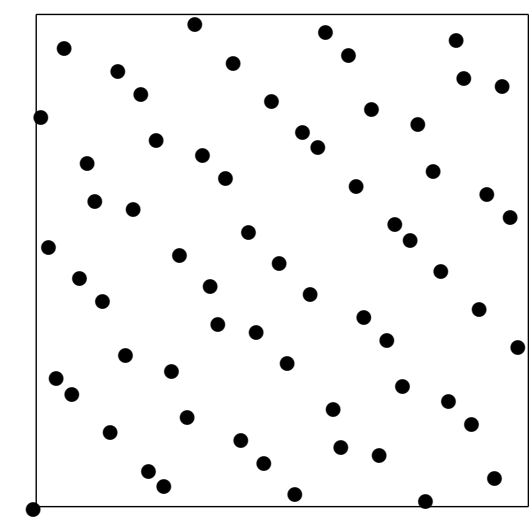

(a) Sobol'

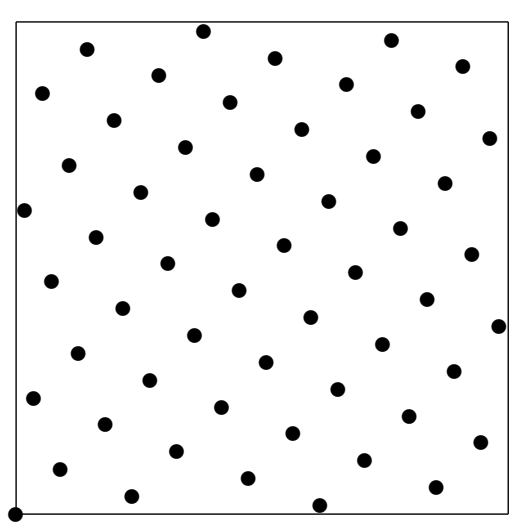

(b) Fibonacci lattice

Deterministic methods have their disadvantages. Although the error is fully deterministic rather than probabilistic, in general the discrepancy bound tends to be far too pessimistic. In other words, QMC methods lack a practical error estimate, whereas the Monte Carlo expected error can easily be obtained by estimating the variance of the function. To overcome this problem, it is recommended that "randomised" QMC methods should be used in practice.

"Shifting" is the simplest form of randomisation. The idea is to move all the points in the same direction by the same amount, and if any point falls outside the unit cube then it is "wrapped" back into the cube from the opposite side. A short description on how to use randomly-shifted QMC methods to provide error estimation is given in the 
Appendix. Another popular but more complicated randomisation method is known as "scrambling". For a survey of randomisation techniques, see Hickernell \& Hong (2002), L'Ecuyer \& Lemieux (2002), and the references therein. Note that shifting preserves the lattice structure while scrambling preserves the net structure: a shifted net may no longer remain a net while a scrambled lattice could cease to be a lattice.

Low-discrepancy sequences such as Sobol' sequences have been widely used for many years by practitioners across various disciplines. In particular, there have been several documented successes for high-dimensional financial derivative calculations such as mortgage-backed securities and option pricing, see e.g. Paskov \& Traub (1995). However, modern analysis and design of digital nets and lattice rules have so far been confined to academic exercises. In many cases these analyses rely on artificial assumptions which are not satisfied in real world problems. Furthermore, interdisciplinary collaborations are needed to properly test these newly developed methods.

Our experiments in this paper will focus on the use of randomly-shifted lattice rules, and as a comparison we consider also Sobol' sequences which are classical examples of digital nets. Unlike most low-discrepancy sequences obtained following some generic schemes, the design of lattice rules requires educated tuning. This might be viewed by some as a disadvantage but others will see it as an opportunity: we have the option and scope to tailor the lattice rule to the integrand. Thus before we can discuss the choice of a lattice rule, we must consider the nature of the integrand.

\subsection{ANOVA Decomposition and Effective Dimension}

It has been observed that the integrands in many practical problems have low effective dimensions. To see what this means, consider for example the simple integrand $f\left(z_{1}, z_{2}, z_{3}, z_{4}\right)=$ $z_{1}+\cos \left(z_{2} z_{3}\right)$ whose nominal dimension is 4 . One could say that the effective dimension is 3 because the integrand depends only on the first three variables. On the other hand, any sensible person would treat this problem as the sum of a one-dimensional integral and a two-dimensional integral, thus concluding that the effective dimension is only 2 .

More generally, every $d$-dimensional function $f$ can be decomposed as a sum of $2^{d}$ terms

$$
f(\mathbf{z})=\sum_{\mathfrak{u} \subseteq\{1, \ldots, d\}} f_{\mathfrak{u}}\left(\mathbf{z}_{\mathfrak{u}}\right)
$$

where $\mathbf{z}_{\mathfrak{u}}$ denotes the set of variables $\left\{z_{j}: j \in \mathfrak{u}\right\}$, and each term $f_{\mathfrak{u}}$ depends only on the set of variables $\mathbf{z}_{\mathfrak{u}}$. This is known as the ANOVA (analysis of variance) decomposition if we impose the condition that $\int_{0}^{1} f_{\mathfrak{u}}\left(\mathbf{z}_{\mathfrak{u}}\right) \mathrm{d} z_{j}=0$ for all $j \in \mathfrak{u}$. It can be shown that the ANOVA terms are orthogonal, i.e. $\int_{[0,1]^{d}} f_{\mathfrak{u}}\left(\mathbf{z}_{\mathfrak{u}}\right) f_{v}\left(\mathbf{z}_{v}\right) \mathrm{d} \mathbf{z}=0$ for all $\mathfrak{u} \neq v$, and they can be expressed recursively by $f_{\mathfrak{u}}\left(\mathbf{z}_{\mathfrak{u}}\right)=\int_{[0,1]^{d-|\mathfrak{u}|}} f(\mathbf{z}) \mathrm{d} \mathbf{z}_{-\mathfrak{u}}-\sum_{v \subset \mathfrak{u}} f_{v}\left(\mathbf{z}_{v}\right)$, where $\mathbf{z}_{-\mathfrak{u}}:=\left\{z_{j}\right.$ : $j \notin \mathfrak{u}\}$. Moreover, we have

$$
\sigma^{2}(f)=\sum_{\mathfrak{u} \subseteq\{1, \ldots, d\}} \sigma^{2}\left(f_{\mathfrak{u}}\right),
$$

that is, the variance of $f$ is the sum of the variances of the ANOVA terms. 
We say that $f_{\mathfrak{u}}$ describes the "interaction" between the variables in $\mathbf{z}_{\mathfrak{u}}$, and we refer to the terms $f_{\mathfrak{u}}$ with $|\mathfrak{u}|=\ell$ collectively as the order- $\ell$ terms. For some functions it may be that only the terms involving say the first 10 variables are important; or on the other hand it may be that all variables are equally important but the higher order interactions are negligible compared with the lower order ones. In both cases these functions are said to have low effective dimensions. Following the definitions in Caflisch, Morokoff \& Owen (1997) and Liu \& Owen (2006),

- the truncation dimension of $f$ is the smallest integer $d_{T}$ such that

$$
\sum_{\mathfrak{u} \subseteq\left\{1, \ldots, d_{T}\right\}} \sigma^{2}\left(f_{\mathfrak{u}}\right) \geq 0.99 \sigma^{2}(f)
$$

- the superposition dimension of $f$ is the smallest integer $d_{S}$ such that

$$
\sum_{|\mathfrak{u}| \leq d_{S}} \sigma^{2}\left(f_{\mathfrak{u}}\right) \geq 0.99 \sigma^{2}(f)
$$

- the mean dimension of $f$ is

$$
d_{M}:=\sum_{\ell=1}^{d}\left(\ell \cdot \frac{1}{\sigma^{2}(f)} \sum_{|\mathfrak{u}|=\ell} \sigma^{2}\left(f_{\mathfrak{u}}\right)\right) .
$$

Thus the truncation dimension corresponds roughly to the number of important variables, while the superposition dimension describes the highest order of significant interactions between variables. The mean dimension is the expected order of $f$, which has a similar interpretation to the superposition dimension.

Note that in general it is impossible to obtain a simple expression for $f_{\mathfrak{u}}$. Fortunately there are ways to compute the effective dimensions of $f$ without having to know the ANOVA terms, see Wang \& Fang (2003) and Liu \& Owen (2006). A brief outline of the techniques is given in the Appendix. Note, however, that these techniques require the evaluation of some integrals involving $f$, and thus the problem of estimating the effective dimensions is at least as hard as the original integration problem.

\subsection{Lattice Rules, Random Shifts, and Weights}

Lattice rules were traditionally used to approximate integrals with periodic integrands. Their role for non-periodic integrands has been known only for the past half decade. Here we give a brief discussion on how to choose a good lattice rule. See Kuo \& Sloan (2005) for a more detailed description of our methodology.

The first step in the modern analysis of lattice rules is to identify a function space $H$ to which the integrand $f$ belongs. In analogy with the ANOVA decomposition, we assume that $f$ belongs to a weighted Sobolev space $H$, in which every function has the decomposition (10) and its variance satisfies (11). The space $H$ contains functions with square-integrable mixed first derivatives, and the inner product of $H$ is "weighted" following the notion first introduced by Sloan \& Woźniakowski (1998). Without going into 
the full details, it suffices to say that we associate a weight, $\gamma_{\mathfrak{u}}$, with every set of variables $\mathbf{z}_{\mathfrak{u}}$, which describes the level of interaction between the variables in $\mathbf{z}_{\mathfrak{u}}$. The $2^{d}$ weights $\gamma_{\mathfrak{u}}$ together model the relative importance between various sets of variables. Roughly speaking, a small $\gamma_{\mathfrak{u}}$ means that the contribution of the ANOVA term $f_{\mathfrak{u}}$ is small compared with the other ANOVA terms of $f$. We set $\gamma_{\emptyset}=1$ to fix the scaling. The limiting case of $\gamma_{\mathfrak{u}}=0$ implies that $f_{\mathfrak{u}}=0$, or in other words, there is no interaction between the variables in $\mathbf{z}_{\mathfrak{u}}$.

To have a function space that best describes our integrand $f$, we must choose the weights $\gamma_{\mathfrak{u}}$ according to the dimension structure of $f$. This corresponds to the variance allocation of $f$ among its ANOVA terms. To limit our choices, we consider just the following settings (e.g. Sloan, Wang \& Woźniakowski, 2004):

- With product weights, we assume that $\gamma_{\mathfrak{u}}:=\prod_{j \in \mathfrak{u}} \gamma_{\{j\}}$, that is, the weight associated with the set of variables $\mathbf{z}_{\mathfrak{u}}$ is automatically assigned the product of the weights for each individual variable $z_{j}$ in this set. This setting is useful when the truncation dimension is low.

- With order-dependent weights, we assume that $\gamma_{\mathfrak{u}}:=\Gamma_{|\mathfrak{u}|}$, that is, the weight associated with the set of variables $\mathbf{z}_{\mathfrak{u}}$ depends only on the cardinality of $\mathfrak{u}$. This setting is useful when the superposition dimension is low but the truncation dimension is still high.

- We can also have finite-order weights by setting $\gamma_{\mathfrak{u}}=0$ for all $|\mathfrak{u}|>q$, with some fixed number $q<d$. Many high-dimensional integrals in practice do appear to have a finite order of just 2 or 3 .

Once we have determined the weights for our function space $H$, we study the worstcase error defined by

$$
e_{N}\left(\xi_{1}, \ldots, \xi_{N}\right):=\sup _{\|f\|_{H} \leq 1}\left|I f-Q_{N} f\right| .
$$

From this definition, we see that $\left|I f-Q_{N} f\right| \leq e_{N}\left(\xi_{1}, \ldots, \xi_{n}\right)\|f\|_{H}$. Thus the worst-case error can be thought of as a type of discrepancy measure. In general we do not have a computable expression for the worst-case error, except when the function space $H$ is a reproducing kernel Hilbert space; this is indeed the case for the Sobolev space.

The worst-case error is then used as our search criterion in a component-by-component algorithm (e.g. Sloan, Kuo \& Joe, 2002) which constructs a good generating vector $\boldsymbol{\eta}$ for randomly-shifted lattice rules. In a nutshell, this is a greedy algorithm which selects the best choice for each component of $\boldsymbol{\eta}$, one at a time, while holding all previously chosen components fixed. There are many variants of this algorithm, including those which aim for good embedded or extensible lattice rules (e.g. Cools, Kuo \& Nuyens, 2006; Dick, Pillichshammer \& Waterhouse, 2006). With a clever implementation, these algorithms can produce, in a very short computational time, good lattice rules with thousands of dimensions and millions of points that achieve close to $\mathcal{O}\left(N^{-1}\right)$ convergence. An outline of the most basic component-by-component algorithm is given in the Appendix. 
Unfortunately, we shall see in the next section that the transformed integrands arising from the log-likelihood integrals do not belong to the weighted Sobolev spaces. This is because we get either functions which are unbounded near the boundary of the unit cube, or functions whose derivatives near the boundary are unbounded and not squareintegrable. In other words, the nice theory discussed above cannot strictly be applied. (In fact, even though there are numerous empirical results showing that QMC methods work well for many finance problems, there has been no concrete theoretical justification because the transformed integrands do not lie in any of the theoretical function space settings.) Having said that, very recently Kuo, Wasilkowski \& Waterhouse (2006) introduced a new function space setting which includes the transformed integrands in the next section. Even though they were only able to prove a $\mathcal{O}\left(N^{-1 / 2}\right)$ convergence for randomly-shifted lattice rules, this is still one big step towards having an applicable theory. Furthermore, it provides some sort of theoretical explanation for the common observation that "QMC always performs no worse than $\mathrm{MC}^{\prime}$.

\section{QMC for Log-likelihood Integrals}

Recall from (2) that our log-likelihood is given by

$$
\ell(\boldsymbol{\beta}, \boldsymbol{\Sigma})=\log \int_{\mathbb{R}^{d}} \exp \{F(\mathbf{w})\} \mathrm{d} \mathbf{w}-\frac{1}{2} \log |\boldsymbol{\Sigma}|-\frac{d}{2} \log (2 \pi)+\mathbf{1}^{\top} c(\mathbf{y}),
$$

where

$$
F(\mathbf{w})=\mathbf{y}^{\top}(\mathbf{X} \boldsymbol{\beta}+\mathbf{W} \mathbf{w})-\mathbf{1}^{\top} b(\mathbf{X} \boldsymbol{\beta}+\mathbf{W} \mathbf{w})-\frac{1}{2} \mathbf{w}^{\top} \boldsymbol{\Sigma}^{-1} \mathbf{w},
$$

with $b(x)=\log \left(1+e^{x}\right)$ and $c(x)=0$ in the Bernoulli model, and $b(x)=e^{x}$ and $c(x)=$ $\log (1 / x !)$ in the Poisson model. Assuming for the moment that the parameters $\boldsymbol{\beta}$ and $\boldsymbol{\Sigma}$ are given and fixed, we wish to approximate $\ell(\boldsymbol{\beta}, \boldsymbol{\Sigma})$.

\subsection{Laplace Approximation}

As already discussed briefly in Section 2 , the Laplace method approximates $\ell(\boldsymbol{\beta}, \boldsymbol{\Sigma})$ using a multivariate normal approximation to the integrand $\exp \{F(\mathbf{w})\}$ via a second order Taylor series approximation of $F(\mathbf{w})$ about its stationary point.

More precisely, the gradient and Hessian of $F(\mathbf{w})$ are given by

$$
\begin{aligned}
\nabla F(\mathbf{w}) & =\mathbf{W}^{\top} \mathbf{y}-\mathbf{W}^{\top} b^{\prime}(\mathbf{X} \boldsymbol{\beta}+\mathbf{W} \mathbf{w})-\boldsymbol{\Sigma}^{-1} \mathbf{w} \\
\nabla^{2} F(\mathbf{w}) & =-\mathbf{W}^{\top} \operatorname{diag}\left(b^{\prime \prime}(\mathbf{X} \boldsymbol{\beta}+\mathbf{W} \mathbf{w})\right) \mathbf{W}-\boldsymbol{\Sigma}^{-1} .
\end{aligned}
$$

We choose $\mathbf{w}^{*}$ and $\Sigma^{*}$ such that

$$
\nabla F\left(\mathbf{w}^{*}\right)=\mathbf{0} \quad \text { and } \quad \mathbf{\Sigma}^{*}=\left(-\nabla^{2} F\left(\mathbf{w}^{*}\right)\right)^{-1},
$$

and we approximate $F(\mathbf{w})$ by

$$
\begin{aligned}
F(\mathbf{w}) & \approx F\left(\mathbf{w}^{*}\right)+\nabla F\left(\mathbf{w}^{*}\right)\left(\mathbf{w}-\mathbf{w}^{*}\right)+\frac{1}{2}\left(\mathbf{w}-\mathbf{w}^{*}\right)^{\top} \nabla^{2} F\left(\mathbf{w}^{*}\right)\left(\mathbf{w}-\mathbf{w}^{*}\right) \\
& =F\left(\mathbf{w}^{*}\right)-\frac{1}{2}\left(\mathbf{w}-\mathbf{w}^{*}\right)^{\top} \mathbf{\Sigma}^{*-1}\left(\mathbf{w}-\mathbf{w}^{*}\right) .
\end{aligned}
$$


Thus

$$
\int_{\mathbb{R}^{d}} \exp \{F(\mathbf{w})\} \mathrm{d} \mathbf{w} \approx(2 \pi)^{d / 2}\left|\Sigma^{*}\right|^{1 / 2} \exp \left\{F\left(\mathbf{w}^{*}\right)\right\}
$$

which leads to

$$
\ell(\boldsymbol{\beta}, \boldsymbol{\Sigma}) \stackrel{\text { Laplace }}{\approx} F\left(\mathbf{w}^{*}\right)+\frac{1}{2} \log \left|\boldsymbol{\Sigma}^{*}\right|-\frac{1}{2} \log |\boldsymbol{\Sigma}|+\mathbf{1}^{\top} c(\mathbf{y})
$$

\subsection{QMC Approximation}

We now approximate the integral in $\ell(\boldsymbol{\beta}, \boldsymbol{\Sigma})$ using QMC methods. Our first step is to transform this integral into the unit cube. As we have explained in Section 3, this is equivalent to importance sampling for the Monte Carlo method. The transformation plays a crucial role as it controls the feature and dimension structure of our transformed integrand. In other words, the transformation determines the difficulty of the integration problem over the unit cube.

The transformation process comprises two stages:

1. Translate and re-centre the mode, and rotate and re-scale the axes.

2. Map the integral into the unit cube.

The first stage helps to eliminate spiky integrands and integrands with support far away from the origin in $\mathbb{R}^{d}$. This can be achieved by a change of variables

$$
\mathbf{v}=\mathbf{P}^{-1}(\mathbf{w}-\boldsymbol{\mu})
$$

with a suitably chosen centre $\boldsymbol{\mu}$ and an invertible matrix $\mathbf{P}$ so that the components of $\mathbf{v}$ are properly scaled with respect to each other. The second stage is to map every integration variable $v_{j}$ from $\mathbb{R}$ into $[0,1]$ using the same cumulative distribution function, that is, we use the substitution

$$
\mathbf{z}=\Psi(\mathbf{v}):=\left(\Psi\left(v_{1}\right), \ldots, \Psi\left(v_{d}\right)\right)^{\top},
$$

where $\Psi$ is the cumulative distribution function of a univariate probability distribution $\psi$. These two stages of the transformation process lead to

$$
\begin{aligned}
\int_{\mathbb{R}^{d}} \exp \{F(\mathbf{w})\} \mathrm{d} \mathbf{w} & =|\mathbf{P}| \int_{\mathbb{R}^{d}} \exp \{F(\boldsymbol{\mu}+\mathbf{P} \mathbf{v})\} \mathrm{d} \mathbf{v} \\
& =|\mathbf{P}| \int_{\mathbb{R}^{d}} \exp \{F(\boldsymbol{\mu}+\mathbf{P} \mathbf{v})\} \prod_{j=1}^{d} \frac{1}{\psi\left(v_{j}\right)} \times \prod_{j=1}^{d} \psi\left(v_{j}\right) \mathrm{d} \mathbf{v} \\
& =|\mathbf{P}| \int_{[0,1]^{d}} \exp \left\{F\left(\boldsymbol{\mu}+\mathbf{P} \Psi^{-1}(\mathbf{z})\right)\right\} \prod_{j=1}^{d} \frac{1}{\psi\left(\Psi^{-1}\left(z_{j}\right)\right)} \mathrm{d} \mathbf{z}
\end{aligned}
$$

where $\Psi^{-1}(\mathbf{z}):=\left(\Psi^{-1}\left(z_{1}\right), \ldots, \Psi^{-1}\left(z_{d}\right)\right)^{\top}$. Hence the transformed integrand is

$$
f(\mathbf{z}):=|\mathbf{P}| \exp \left\{F\left(\boldsymbol{\mu}+\mathbf{P} \Psi^{-1}(\mathbf{z})\right)\right\} \prod_{j=1}^{d} \frac{1}{\psi\left(\Psi^{-1}\left(z_{j}\right)\right)},
$$


and a QMC rule with points $\xi_{1}, \ldots, \xi_{N}$ gives the approximation

$$
\ell(\boldsymbol{\beta}, \boldsymbol{\Sigma}) \stackrel{\mathrm{QMC}}{\approx} \log \left(\frac{1}{N} \sum_{i=1}^{N} f\left(\boldsymbol{\xi}_{i}\right)\right)-\frac{1}{2} \log |\boldsymbol{\Sigma}|-\frac{d}{2} \log (2 \pi)+\mathbf{1}^{\top} c(\mathbf{y}) .
$$

How should we choose the pair $\boldsymbol{\mu}$ and $\mathbf{P}$ ? Clearly we should re-centre our integrand based on the stationary point $\mathbf{w}^{*}$ of $F(\mathbf{w})$. On the other hand, the Laplace method provides a fairly good approximation locally around $\mathrm{w}^{*}$, and we can think of $\Sigma^{*}$ as being chosen to match the curvature of the integrand at $\mathrm{w}^{*}$. This motivates the choice

$$
\boldsymbol{\mu}=\mathbf{w}^{*} \quad \text { and } \quad \mathbf{P}=\mathbf{A}^{*},
$$

where $\mathbf{A}^{*}$ is a matrix satisfying

$$
\Sigma^{*}=\mathbf{A}^{*} \mathbf{A}^{* \top} \text {. }
$$

An obvious choice is to take $\mathbf{A}^{*}$ to be the Cholesky factor of $\boldsymbol{\Sigma}^{*}$. Alternatively, $\mathbf{A}^{*}$ can be obtained from spectral decomposition as $\left(\sqrt{\lambda_{1}} \nu_{1}, \ldots, \sqrt{\lambda_{d}} \boldsymbol{\nu}_{d}\right)$, where $\lambda_{1} \geq \cdots \geq \lambda_{d}$ are the eigenvalues of $\Sigma^{*}$ and $\boldsymbol{\nu}_{1}, \ldots, \boldsymbol{\nu}_{d}$ are the corresponding unit column eigenvectors. This second choice of decomposition is often associated with the term principal component analysis (PCA) in the QMC community even though all the eigenvalues and eigenvectors are used. We shall refer to this second choice of $\mathbf{A}^{*}$ as the PCA factor of $\boldsymbol{\Sigma}^{*}$.

Note that we could also take $\mathbf{P}=\mathbf{A}$, with $\mathbf{A}$ being the Cholesky or PCA factor of the covariance matrix $\Sigma$ from the normal distribution already present in the integrand. However, exploratory calculations indicate that such a transformation leads to very poor results. The bottom line is that $\Sigma$ simply does not capture the feature of the integrand around $\mathbf{w}^{*}$.

Having chosen $\boldsymbol{\mu}$ and $\mathbf{P}$ for the first stage of the transformation process, we now choose a probability distribution $\psi$ for the second stage.

Transformation 1 The most intuitive choice is to take $\psi$ to be the standard normal distribution,

$$
\psi(v)=(2 \pi)^{-1 / 2} \exp \left(-\frac{1}{2} v^{2}\right) .
$$

The transformed integrand is then

$$
f_{1}(\mathbf{z}):=(2 \pi)^{d / 2}\left|\Sigma^{*}\right|^{1 / 2} \exp \left\{F\left(\mathbf{w}^{*}+\mathbf{A}^{*} \Psi^{-1}(\mathbf{z})\right)+\frac{1}{2} \Psi^{-1}(\mathbf{z})^{\top} \Psi^{-1}(\mathbf{z})\right\} .
$$

Note that there is no closed form expression for $\Psi^{-1}$, although computational techniques based on rational approximation are well known.

Observe that $f_{1}$ is continuous on $(0,1)^{d}$, but it could be unbounded near the boundaries of the unit cube. Using the definition of $\Sigma^{*}$ we can write the exponent in $f_{1}$ as

$$
\begin{aligned}
& \mathbf{y}^{\top}\left(\mathbf{X} \boldsymbol{\beta}+\mathbf{W w}^{*}+\mathbf{W A}^{*} \Psi^{-1}(\mathbf{z})\right)-\mathbf{1}^{\top} b\left(\mathbf{X} \boldsymbol{\beta}+\mathbf{W w}^{*}+\mathbf{W A}^{*} \Psi^{-1}(\mathbf{z})\right) \\
& -\frac{1}{2} \mathbf{w}^{* \top} \boldsymbol{\Sigma}^{-1} \mathbf{w}^{*}-\mathbf{w}^{* \top} \boldsymbol{\Sigma}^{-1} \mathbf{A}^{*} \Psi^{-1}(\mathbf{z}) \\
& +\frac{1}{2}\left(\mathbf{W A}^{*} \Psi^{-1}(\mathbf{z})\right)^{\top} \operatorname{diag}\left(b^{\prime \prime}\left(\mathbf{X} \boldsymbol{\beta}+\mathbf{W} \mathbf{w}^{*}\right)\right)\left(\mathbf{W A}^{*} \Psi^{-1}(\mathbf{z})\right) .
\end{aligned}
$$

When $b(x)=\log \left(1+e^{x}\right)$, the positive quadratic term in the exponent dominates. Thus $f_{1}$ is unbounded at all boundaries of the cube. Consider now $b(x)=e^{x}$. Then the exponent 
is a balance between negative exponential and positive quadratic terms, and it could potentially approach $\pm \infty$. Thus in this case $f_{1}$ is unbounded at some boundaries of the cube. A more detailed analysis of the boundary behaviour for the latter case is given in the Appendix.

The transformation described above can lead to integrands which are unbounded at the boundaries of the unit cube. However, unbounded integrands do not belong to the Sobolev spaces discussed in Section 3, and therefore the existing theory on lattice rules cannot strictly be applied. Our aim is to find an alternative transformation such that the transformed integrand is bounded everywhere on $[0,1]^{d}$.

Transformation 2 Instead of using the normal distribution to map the integral into the unit cube, we use the logistic distribution

$$
\psi(v)=\frac{e^{v / \lambda}}{\lambda\left(1+e^{v / \lambda}\right)^{2}} .
$$

This distribution has a bell shape, but its tails only have exponential decay. The cumulative distribution function is

$$
\Psi(v)=\frac{e^{v / \lambda}}{1+e^{v / \lambda}}, \quad \text { with } \quad \Psi^{-1}(z)=\lambda \log \left(\frac{z}{1-z}\right) .
$$

This inverse function is known as the logit function or the logistic map. The transformed integrand in this case is

$$
f_{2}(\mathbf{z}):=\lambda^{d}\left|\mathbf{\Sigma}^{*}\right|^{1 / 2} \exp \left\{F\left(\mathbf{w}^{*}+\mathbf{A}^{*} \Psi^{-1}(\mathbf{z})\right)\right\} \prod_{j=1}^{d}\left(e^{-\Psi^{-1}\left(z_{j}\right) / \lambda}+2+e^{\Psi^{-1}\left(z_{j}\right) / \lambda}\right) .
$$

As before we consider the boundary behaviour of $f_{2}$. After grouping the product term into the exponential function, the exponent is

$$
\begin{aligned}
& \mathbf{y}^{\top}\left(\mathbf{X} \boldsymbol{\beta}+\mathbf{W}\left(\mathbf{w}^{*}+\mathbf{A}^{*} \Psi^{-1}(\mathbf{z})\right)\right)-\mathbf{1}^{\top} b\left(\mathbf{X} \boldsymbol{\beta}+\mathbf{W}\left(\mathbf{w}^{*}+\mathbf{A}^{*} \Psi^{-1}(\mathbf{z})\right)\right) \\
& -\frac{1}{2}\left(\mathbf{w}^{*}+\mathbf{A}^{*} \Psi^{-1}(\mathbf{z})\right)^{\top} \boldsymbol{\Sigma}^{-1}\left(\mathbf{w}^{*}+\mathbf{A}^{*} \Psi^{-1}(\mathbf{z})\right)+\sum_{j=1}^{d} \log \left(e^{-\Psi^{-1}\left(z_{j}\right) / \lambda}+2+e^{\Psi^{-1}\left(z_{j}\right) / \lambda}\right) .
\end{aligned}
$$

When $b(x)=\log \left(1+e^{x}\right)$, the negative quadratic term dominates the exponent and so $f_{2}$ is bounded everywhere on $[0,1]^{d}$. If $b(x)=e^{x}$, then the exponent is dominated by negative exponential as well as negative quadratic terms, and it could only approach $-\infty$ and not $+\infty$. Thus in this case $f_{2}$ is also bounded everywhere on $[0,1]^{d}$.

It is worth noting that even though the integrands are bounded, the derivatives can be huge. In practice it may actually be easier to handle an unbounded integrand with weak singularities rather than bounded integrands with huge boundary derivatives.

We could also consider other probability distributions for the second stage of the transformation. One other possible choice is to use a skewed normal distribution that has different standard deviations for positive and negative values. Since our integrand is skewed in some way, this transformation may prove to be advantageous. 


\section{Numerical Experiments}

In our experiments here we consider two simplified problems in which we assumed that the parameters are given and fixed. The goal here is to find the best transformation to obtain good QMC approximations of the log-likelihood integrals. In the next section we will embed these integral calculations in an optimisation procedure to find the optimal parameter set.

Example 1 We consider a simple parameter driven Poisson state-space model where $\mathbf{w}=$ $\left(w_{1}, \ldots, w_{d}\right)^{\top}$ is an auto-regression of degree 1 (i.e., $w_{j}=\phi w_{j-1}+\eta_{j}$ with $\eta_{j}$ i.i.d. $\left.N\left(0, \sigma^{2}\right)\right)$. Here $b(x)=e^{x}, c(x)=\log (1 / x !), \mathbf{W}=\mathbf{I}, \mathbf{X}=\mathbf{1}, \boldsymbol{\beta}=\beta$, and the covariance matrix is Toeplitz

$$
\Sigma=\frac{\sigma^{2}}{1-\phi^{2}}\left[\begin{array}{cccccc}
1 & \phi & \phi^{2} & \cdots & \phi^{d-2} & \phi^{d-1} \\
\phi & 1 & \phi & \cdots & \phi^{d-3} & \phi^{d-2} \\
\phi^{2} & \phi & 1 & \cdots & \phi^{d-4} & \phi^{d-3} \\
\vdots & \vdots & \vdots & \ddots & \vdots & \vdots \\
\phi^{d-2} & \phi^{d-3} & \phi^{d-4} & \cdots & 1 & \phi \\
\phi^{d-1} & \phi^{d-2} & \phi^{d-3} & \cdots & \phi & 1
\end{array}\right], \quad \text { with } \quad|\boldsymbol{\Sigma}|=\frac{\sigma^{2 d}}{1-\phi^{2}} .
$$

The Cholesky factor of $\boldsymbol{\Sigma}=\mathbf{A} \mathbf{A}^{T}$ and its inverse are known explicitly

$\mathbf{A}=\sigma\left[\begin{array}{ccccc}\frac{1}{\sqrt{1-\phi^{2}}} & 0 & 0 & \cdots & 0 \\ \frac{\phi}{\sqrt{1-\phi^{2}}} & 1 & 0 & \cdots & 0 \\ \frac{\phi^{2}}{\sqrt{1-\phi^{2}}} & \phi & 1 & \cdots & 0 \\ \vdots & \vdots & \vdots & \ddots & \vdots \\ \frac{\phi^{d-1}}{\sqrt{1-\phi^{2}}} & \phi^{d-2} & \phi^{d-3} & \cdots & 1\end{array}\right]$ and $\mathbf{A}^{-1}=\frac{1}{\sigma}\left[\begin{array}{cccccc}\sqrt{1-\phi^{2}} & 0 & 0 & \cdots & 0 & 0 \\ -\phi & 1 & 0 & \cdots & 0 & 0 \\ 0 & -\phi & 1 & \cdots & 0 & 0 \\ \vdots & \vdots & \vdots & \ddots & \vdots & \\ 0 & 0 & 0 & \cdots & -\phi & 1\end{array}\right]$.

The inverse of $\Sigma$ is tridiagonal

$$
\Sigma^{-1}=\frac{1}{\sigma^{2}}\left[\begin{array}{cccccc}
1 & -\phi & 0 & \cdots & 0 & 0 \\
-\phi & 1+\phi^{2} & -\phi & \cdots & 0 & 0 \\
0 & -\phi & 1+\phi^{2} & \cdots & 0 & 0 \\
\vdots & \vdots & \vdots & \ddots & \vdots & \vdots \\
0 & 0 & 0 & \cdots & 1+\phi^{2} & -\phi \\
0 & 0 & 0 & \cdots & -\phi & 1
\end{array}\right] .
$$

We have a data file containing count data $\mathbf{y}=\left(y_{1}, \ldots, y_{d}\right)^{\top}$ up to dimension $d=200$. These data were simulated from the parameters $\beta=0.7, \sigma^{2}=0.3$ and $\phi=0.5$. Due to the time-series structure, we can consider this integration problem with $d$ taking any value up to 200 . We shall consider $d=25,50,100,150,200$.

For this example we have

$$
\begin{aligned}
F(\mathbf{w}) & =\sum_{j=1}^{d}\left(\beta y_{j}+w_{j} y_{j}-e^{\beta} e^{w_{j}}\right)-\frac{1}{2} \mathbf{w}^{\top} \mathbf{\Sigma}^{-1} \mathbf{w}, \\
\nabla F(\mathbf{w}) & =\mathbf{y}-e^{\beta} e^{\mathbf{w}}-\mathbf{\Sigma}^{-1} \mathbf{w} \\
\nabla^{2} F(\mathbf{w}) & =-\operatorname{diag}\left(e^{\beta} e^{\mathbf{w}}\right)-\mathbf{\Sigma}^{-1} .
\end{aligned}
$$


To obtain the stationary point $\mathbf{w}^{*}$ satisfying $\nabla F\left(\mathbf{w}^{*}\right)=\mathbf{0}$, we use the Newton iteration

$$
\left(-\nabla^{2} F\left(\mathbf{w}^{(k)}\right)\right)\left(\mathbf{w}^{(k+1)}-\mathbf{w}^{(k)}\right)=\nabla F\left(\mathbf{w}^{(k)}\right),
$$

starting at $\mathbf{w}^{(0)}=\mathbf{0}$. Each iteration can be solved by a Cholesky factorisation of $\left(-\nabla^{2} F\left(\mathbf{w}^{(k)}\right)\right)$ followed by forward and back substitutions, see the LAPACK function DPOTRS (Anderson et al., 1999). Once a satisfactory $\mathbf{w}^{*}$ is found, the matrix $\Sigma^{*}$ discussed in Section 4.1 is given by $\Sigma^{*}=\left(-\nabla^{2} F\left(\mathbf{w}^{*}\right)\right)^{-1}$, where the inverse matrix can be obtained by making use of the Cholesky factorisation, see the LAPACK function DPOTRI.

$\#$

Example 2 We consider a generalised linear mixed model which is a simple semi-parametric regression model with Poisson response. In this example $b(x)=e^{x}, c(x)=\log (1 / x !)$, $\mathbf{w}=\mathbf{u}=\left(u_{1}, \ldots, u_{d}\right)^{\top}, \mathbf{y}=\left(y_{1}, \ldots, y_{n}\right)^{\top} \in\{0,1,2, \ldots\}^{n}$, and

$$
\mathbf{X}=\left[\begin{array}{ccc}
1 & x_{11} & x_{21} \\
1 & x_{12} & x_{22} \\
\vdots & \vdots & \vdots \\
1 & x_{1 n} & x_{2 n}
\end{array}\right], \quad \boldsymbol{\beta}=\left[\begin{array}{c}
\beta_{0} \\
\beta_{1} \\
\beta_{2}
\end{array}\right], \quad \mathbf{W}=\mathbf{Z}=\left[\left(x_{2 i}-\kappa_{j}\right)_{+}\right]_{\substack{1 \leq i \leq n \\
1 \leq j \leq d}},
$$

where $x_{11}, \ldots, x_{1 n} \in\{0,1\}, x_{21}, \ldots, x_{2 n} \in(0,1)$, and $\kappa_{1}, \ldots, \kappa_{d} \in(0,1)$ are fixed numbers ranging between the maximum and minimum of $x_{2 i}$, known as knots. The covariance matrix is $\boldsymbol{\Sigma}=\sigma^{2} \mathbf{I}$.

We have a simulated data set of $\mathbf{x}_{1}, \mathbf{x}_{2}, \mathbf{y}$, and numbers $\kappa_{j}$, for $d=25$ and $n=$ 500. (Note that the dimension for this example is fixed.) A good starting point for the parameters is given by $\beta_{0}=1, \beta_{1}=0.77, \beta_{2}=1.5$, and $\sigma^{2}=51$.

For this example we have

$$
\begin{aligned}
F(\mathbf{u}) & =\mathbf{y}^{\top}(\mathbf{X} \boldsymbol{\beta}+\mathbf{Z u})-\mathbf{1}^{\top} \exp (\mathbf{X} \boldsymbol{\beta}+\mathbf{Z u})-\mathbf{u}^{\top} \mathbf{u} /\left(2 \sigma^{2}\right) \\
\nabla F(\mathbf{u}) & =\mathbf{Z}^{\top}(\mathbf{y}-\exp (\mathbf{X} \boldsymbol{\beta}+\mathbf{Z u}))-\mathbf{u} / \sigma^{2} \\
\nabla^{2} F(\mathbf{u}) & =-\mathbf{Z}^{\top} \operatorname{diag}(\exp (\mathbf{X} \boldsymbol{\beta}+\mathbf{Z u})) \mathbf{Z}-\left(1 / \sigma^{2}\right) \mathbf{I} .
\end{aligned}
$$

The stationary point $\mathbf{u}^{*}$ and the matrix $\Sigma^{*}$ can be obtained in the same way as in Example 1.

Following Subsection 4.2, we consider four transformations: $\psi$ is either the standard normal distribution or the logistic distribution with $\lambda=0.6$, and $\mathbf{A}^{*}$ is either the Cholesky factor or the PCA factor of $\Sigma^{*}$. For convenience we shall refer to these four transformation methods as "Normal + PCA", "Normal + Cholesky", "Logistic 0.6 + PCA", and "Logistic $0.6+$ Cholesky".

\subsection{Effective Dimension Estimation}

To gain some insights to the dimension structure of these transformed integrands in the unit cube, we compute their effective dimensions. Figure 3 shows the estimated truncation dimension and mean dimension for both examples under the four transformations described above. (More details regarding how these numbers were obtained are given in the Appendix.) 
Figure 3: Effective dimensions of transformed integrands.
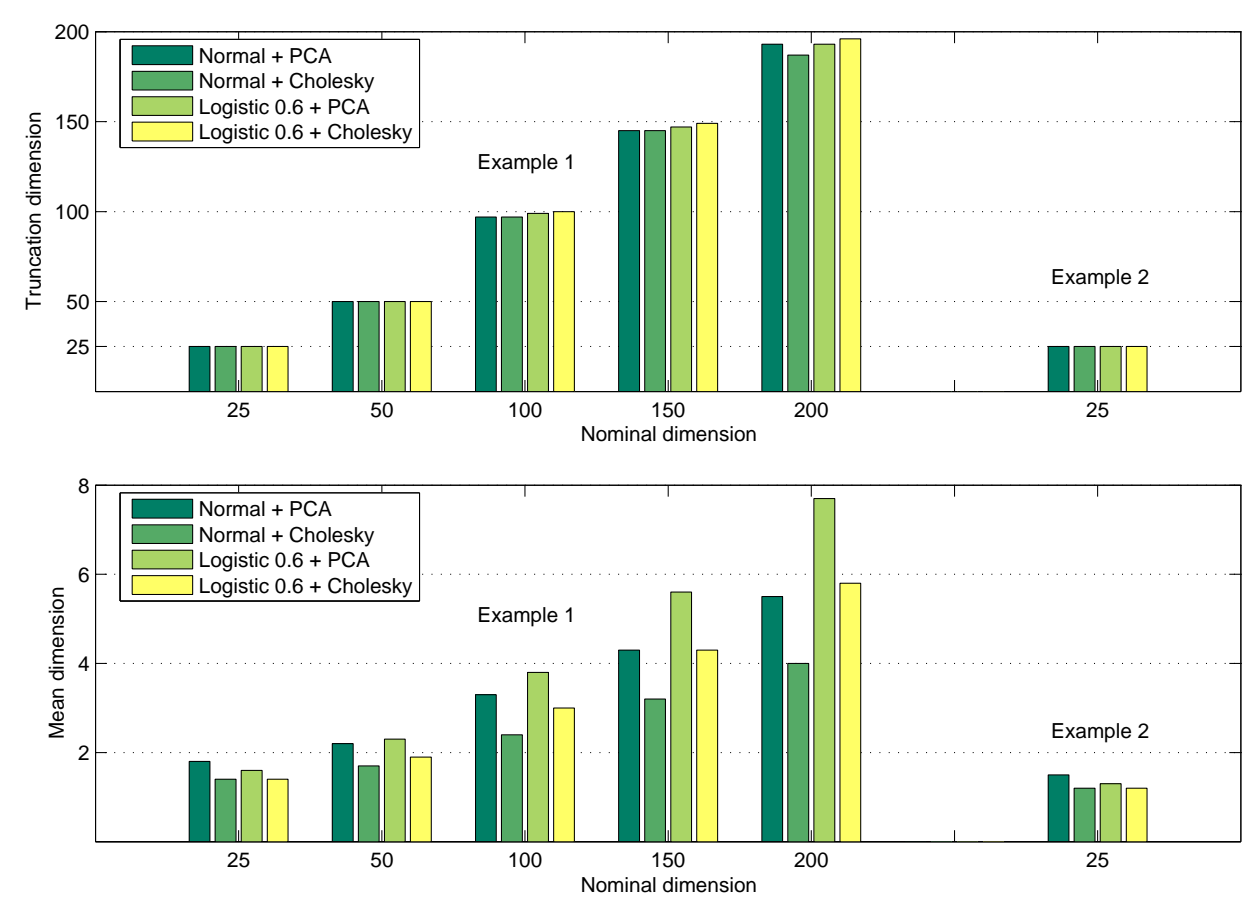

It is important to realise that these numbers can only be used a rough guide because the errors in these estimates can be huge. The superposition dimension estimates are the most unreliable of all and this is why we do not present the results here. (Past experiences indicate that the mean dimensions tend to be under-estimates of the superposition dimensions.) Having said that, we do get a clear picture of the dimension structure of these functions: the truncation dimension is essentially the nominal dimension regardless of the transformation method. Unlike many option pricing problems in finance, the PCA decomposition does not help to reduce the truncation dimension for our examples here.

On the other hands, the transformation method appears to have some small effect on the mean dimension. Although no concrete conclusions can be drawn from these results, the PCA decomposition generally leads to higher mean dimension than the Cholesky decomposition does. As the nominal dimension increases, the mean dimension (and most likely the superposition dimension too) increases rapidly, indicating that these highdimensional problems are truly high-dimensional. This is again contrary to many finance problems in which the mean dimension remains around 2 as the nominal dimension increases.

\subsection{Two-dimensional Projections}

We produce plots of some two-dimensional projections of these transformed integrands to get an idea of their boundary behaviour. A number of selected plots are included in Figures 4 and 5. 
Figure 4: Two-dimensional projections of transformed integrand obtained using (a) normal distribution and (b) logistic distribution with $\lambda=0.6$.

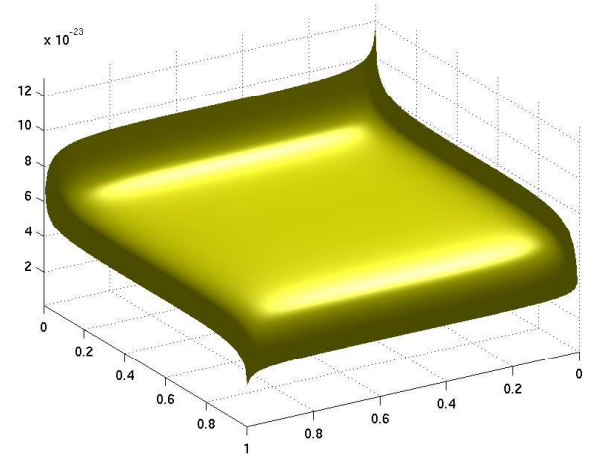

(a.1)

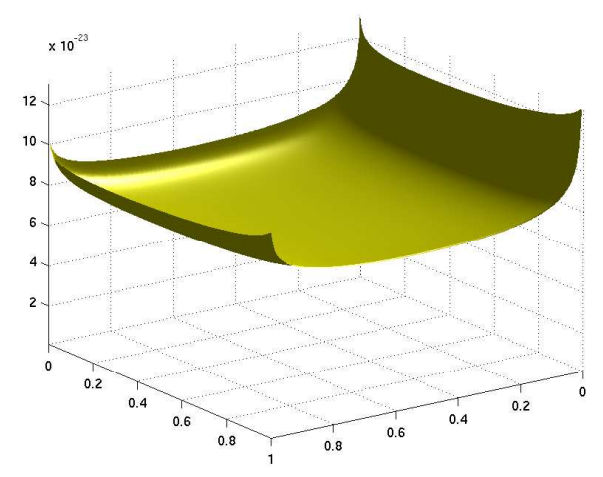

(a.2)

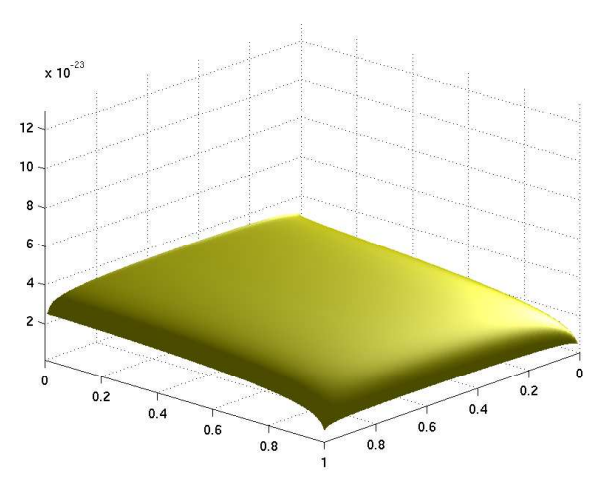

(a.3)

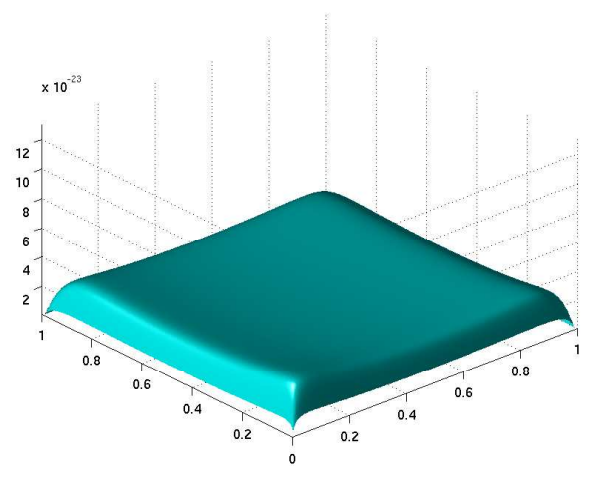

(b.1)

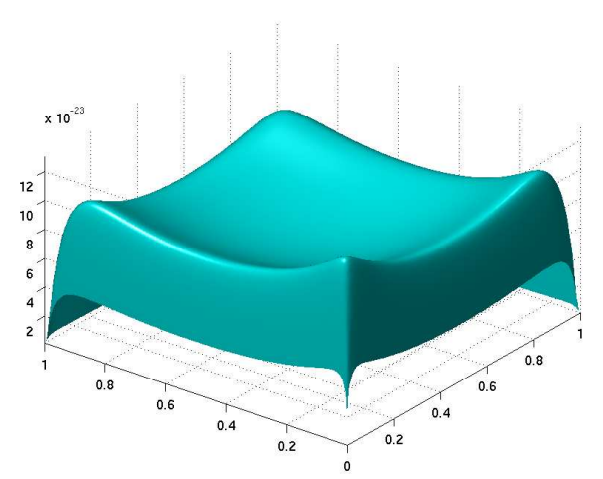

(b.2)

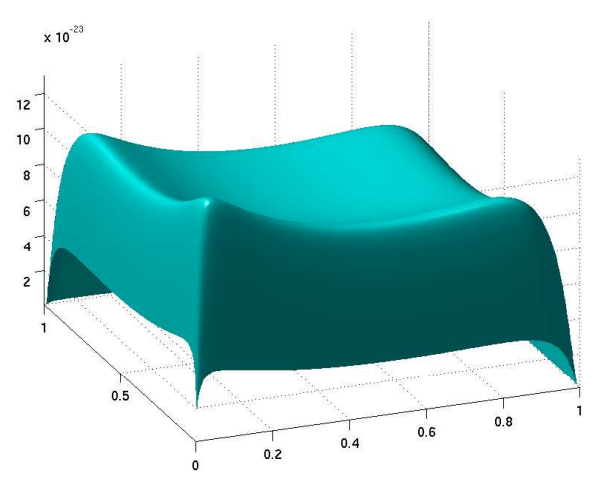

(b.3) 
Figure 5: Two-dimensional projections of transformed integrand obtained (c) without centring and rescaling and $(\mathrm{d})$ with centring but without rescaling.

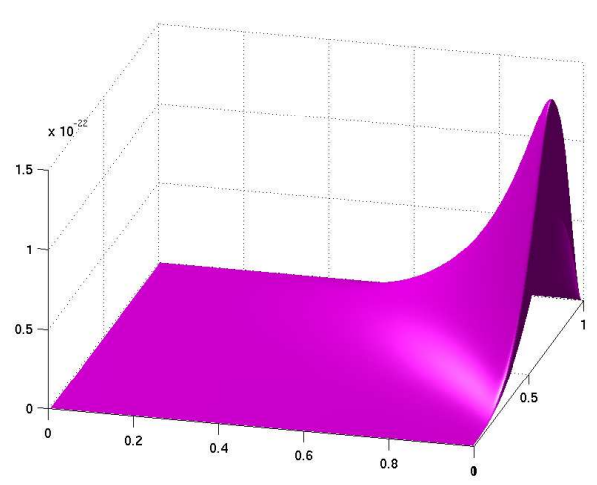

(c)

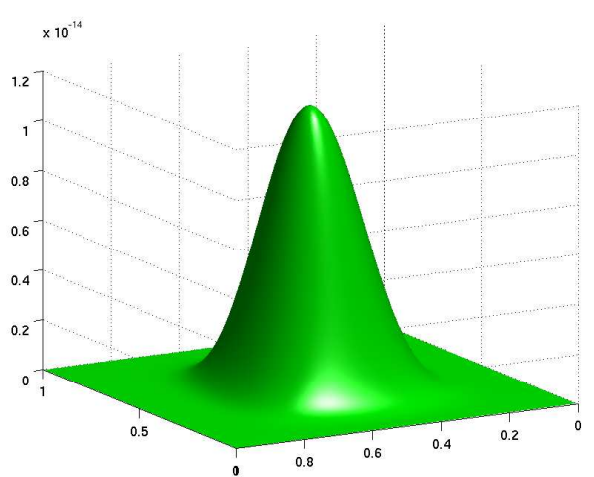

(d)

Figure 4 shows the general characteristics of these transformed integrands by means of their two-dimensional projections. For example, the graph (a.1) corresponds to the transformed integrand obtained by using "Normal + PCA" in Example 1 with $d=25$. It shows a function of the first and the fifth variables, with all other variables fixed the value 0.5 . In other words, it is a two-dimensional projection of the transformed integrand from the centre of the unit cube and parallel to the axes $z_{1}$ and $z_{5}$. The graph suggests that the function goes to infinity when either variable goes to 0 , but it is otherwise fairly flat. The graphs (a.2) and (a.3) show other projections of the same transformed integrand in different dimensions and anchored at different positions in the unit cube. In fact, for both Examples 1 and 2, the transformations based on the standard normal distribution lead to projections like those in (a), and the transformations based on the logistic distribution lead to projections like those in (b). This is regardless of whether PCA or Cholesky decomposition is used. These features are as we have predicted: the use of standard normal distribution can result in unbounded integrands, and the use of logistic distribution always give bounded integrands but the derivatives near the boundary can be huge.

As a comparison, in Figure 5 we present the projections of some badly transformed integrands: (c) shows the support of the integrand off to one corner and (d) shows a narrow spike at the centre. They are associated with transformed integrands obtained without centring, or with centring but without rescaling. We do not expect MC or QMC methods to do well for these badly transformed integrands. (As it turned out, the results were way off.) Further details about these figures are given in the Appendix.

\subsection{Integral Calculation}

Now we compute the integral for each transformed integrand using the Monte Carlo method, the Sobol' sequence, and four lattice rules constructed based on various choices of weights. (See the Appendix for details regarding these lattice rules.) In each calculation, we use 10 random shifts of $N$ points, $N=2^{15}=32768$ or $N=2^{16}=65536$, and we 
estimate the standard errors. We then repeat this process 30 times and produce a comparison of the boxplots for the $\log _{10}$ relative integrals and $\log _{10}$ relative standard errors. The results for Example 1 with $d=25$, Example 2 (which has $d=25$ ), and Example 1 with $d=200$ are presented in Figures 6, 7, and 8, respectively. Note that since the boundary behaviour is the dominating feature of some transformed integrands, it is necessary that we use a good random number generator with double precision (see the Appendix for details).

More precisely, for each of the six integration rules and each of the four transformation methods, we compute

$$
I_{s, k}(\text { rule }, \text { trans })=\frac{1}{N} \sum_{i=1}^{N} f_{s, k, i}(\text { rule }, \text { trans })
$$

for $s=1,2, \ldots, 30$ and $k=1,2, \ldots, 10$, where $f_{s, k, i}$ denotes the transformed integrand value at the $k$ th random shift of the $i$ th integration point during the $s$ th repetition. For each repetition $s=1,2, \ldots, 30$, we compute the estimated integral and the corresponding standard error

$$
\begin{aligned}
\bar{I}_{s}(\text { rule }, \text { trans }) & =\frac{1}{10} \sum_{k=1}^{10} I_{s, k}(\text { rule }, \text { trans }) \\
E_{s}(\text { rule }, \text { trans }) & =\sqrt{\frac{1}{10 \times 9} \sum_{k=1}^{10}\left(I_{s, k}(\text { rule }, \text { trans })-\bar{I}_{s}(\text { rule }, \text { trans })\right)^{2}} .
\end{aligned}
$$

Each boxplot in the figures contains the logarithm (base 10) of a scaled version of 30 numbers, either

$$
\log _{10}\left(\frac{\bar{I}_{s}(\text { rule, trans })}{\operatorname{median}_{1 \leq s \leq 30} \bar{I}_{s}(\mathrm{MC}, \text { Normal }+ \text { PCA })}\right)
$$

or

$$
\log _{10}\left(\frac{E_{s}(\text { rule, trans })}{\operatorname{median}_{1 \leq s \leq 30} \bar{I}_{s}(\mathrm{MC}, \text { Normal + PCA })}\right) .
$$

In other words, all results have been scaled by the median of the MC estimated integral using "Normal + PCA". Thus the first boxplot in every figure should have its black dot exactly at 0 .

\section{Conclusion from Figure 6}

First we discuss the results in Figure 6 for Example 1 with $d=25$ and $N=32768$. At a glance it is clear that QMC dramatically outperforms MC: the integral estimates for both $\mathrm{MC}$ and QMC are unbiased, but the standard errors for MC are significantly larger than those for QMC.

For three of the four transformations, the lattice rule "Lat1" gives noticeably worse results than Sobol' points and the other three lattice rules. This particular lattice rule is designed for integrands with only second-order interactions (assuming no higher order terms are present), and is clearly not suitable for our integrands here. It goes to show that 
Figure 6: Example 1 with $d=25 . \log _{10}$ relative integrals and $\log _{10}$ relative standard errors obtained from 30 repetitions based on 10 random shifts of 32768 points.
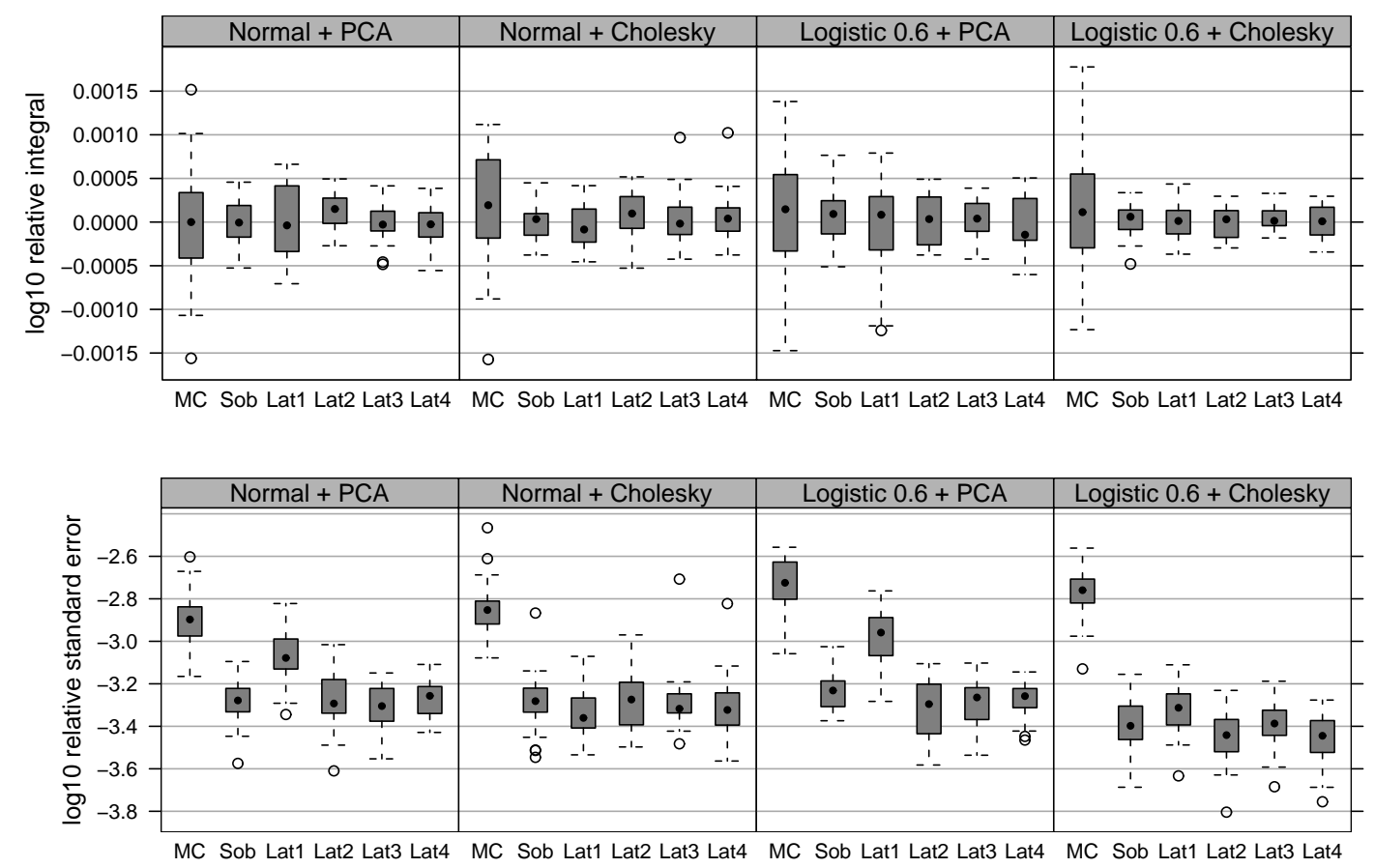

the selection of weights in the design of lattice rules to match the dimension structure of the integrands indeed have some practical effects. Leaving this "bad" lattice rule aside, the other three lattice rules appear to give slightly better results than the Sobol' points.

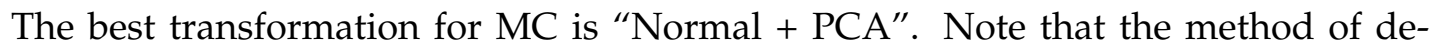
composition (i.e. PCA or Cholesky) should have no impact on the performance of MC theoretically. This is because the MC root mean square error depends on the total variance of the transformed integrand, which is invariant under different decompositions.

The best transformation for QMC is "Logistic 0.6 + Cholesky". Quantitatively, we take the average median of the relative standard errors for QMC (with "Lat1" excluded) under each transformation method and compare it with the median of the relative standard error for MC under its best transformation "Normal + PCA". The improvement of QMC over MC in terms of the reduction in standard errors are 3.3 for "Logistic $0.6+$ Cholesky", 2.5 for "Normal + Cholesky", 2.4 for "Normal + PCA", and 2.3 for "Logistic $0.6+$ PCA". In other words, the MC standard errors can be more than three times the QMC standard errors.

\section{Conclusion from Figure 7}

Figure 7 includes results for Example 2 (which has a fixed dimension $d=25$ ) with $N=$ 32768. For this example, the superiority of QMC over MC stands out even more. It is also very clear that the normal distribution consistently gives better results than the logistic 
Figure 7: Example $2(d=25) \cdot \log _{10}$ relative integrals and $\log _{10}$ relative standard errors obtained from 30 repetitions based on 10 random shifts of 32768 points.
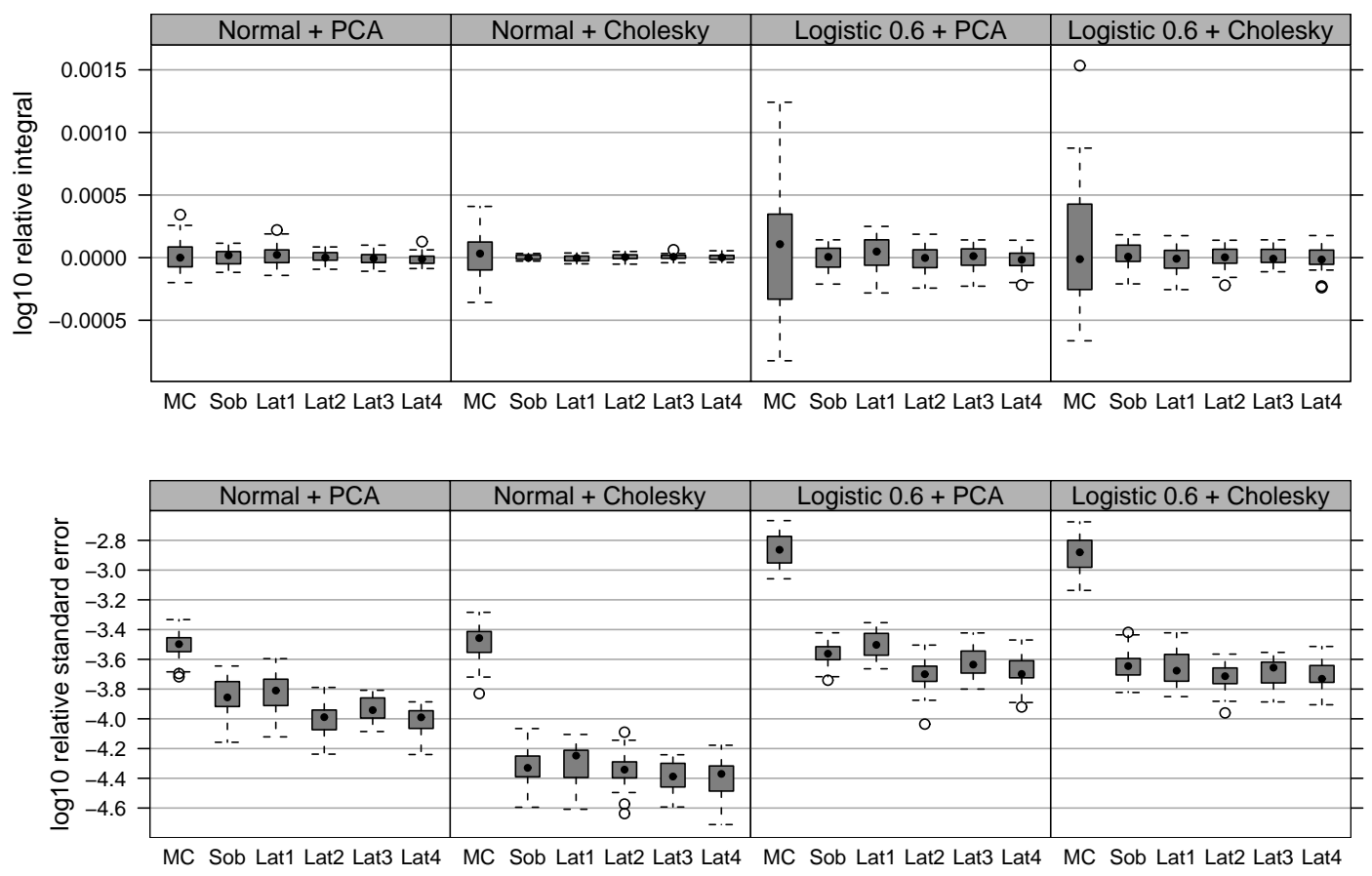

distribution. The best transformation for MC is again "Normal + PCA", but the best transformation for QMC is now "Normal + Cholesky". We experimented with several values of $\lambda$ for the logistic distribution and none of them give better results than the standard normal distribution. There must be some foundational differences between the integrands of Examples 1 and 2 which are yet to be identified.

As in Figure 6, the lattice rule "Lat1" appears to give worse results than other lattice rules, although the differences are not so noticeable here. With "Lat1" excluded, the other three lattice rules again appear to give slightly better results than the Sobol' points. In terms of the reduction in standard errors, QMC beats MC (under its best transformation "Normal + PCA") by a factor of 7.2 for "Normal + Cholesky", 2.8 for "Normal + PCA", 1.5 for "Logistic $0.6+$ Cholesky", and 1.4 for "Logistic $0.6+$ PCA". For this example, the MC standard errors can be more than seven times the QMC standard errors.

\section{Conclusion from Figure 8}

The results we have discussed so far correspond to a low nominal dimension of $d=$ 25. Although $d=25$ is reasonable for a random effect model such as Example 2, the dimension of interest for a time-series model like Example 1 is often in the hundreds or thousands. Therefore we consider $d=50,100,150,200$ in Example 1 and carry out similar calculations as before (but with more integration points). It is noticeable that as the dimension $d$ increases, the superiority of QMC over MC is dramatically reduced. 
Figure 8: Example 1 with $d=200 . \log _{10}$ relative integrals and $\log _{10}$ relative standard errors obtained from 30 repetitions based on 10 random shifts of 65536 points.
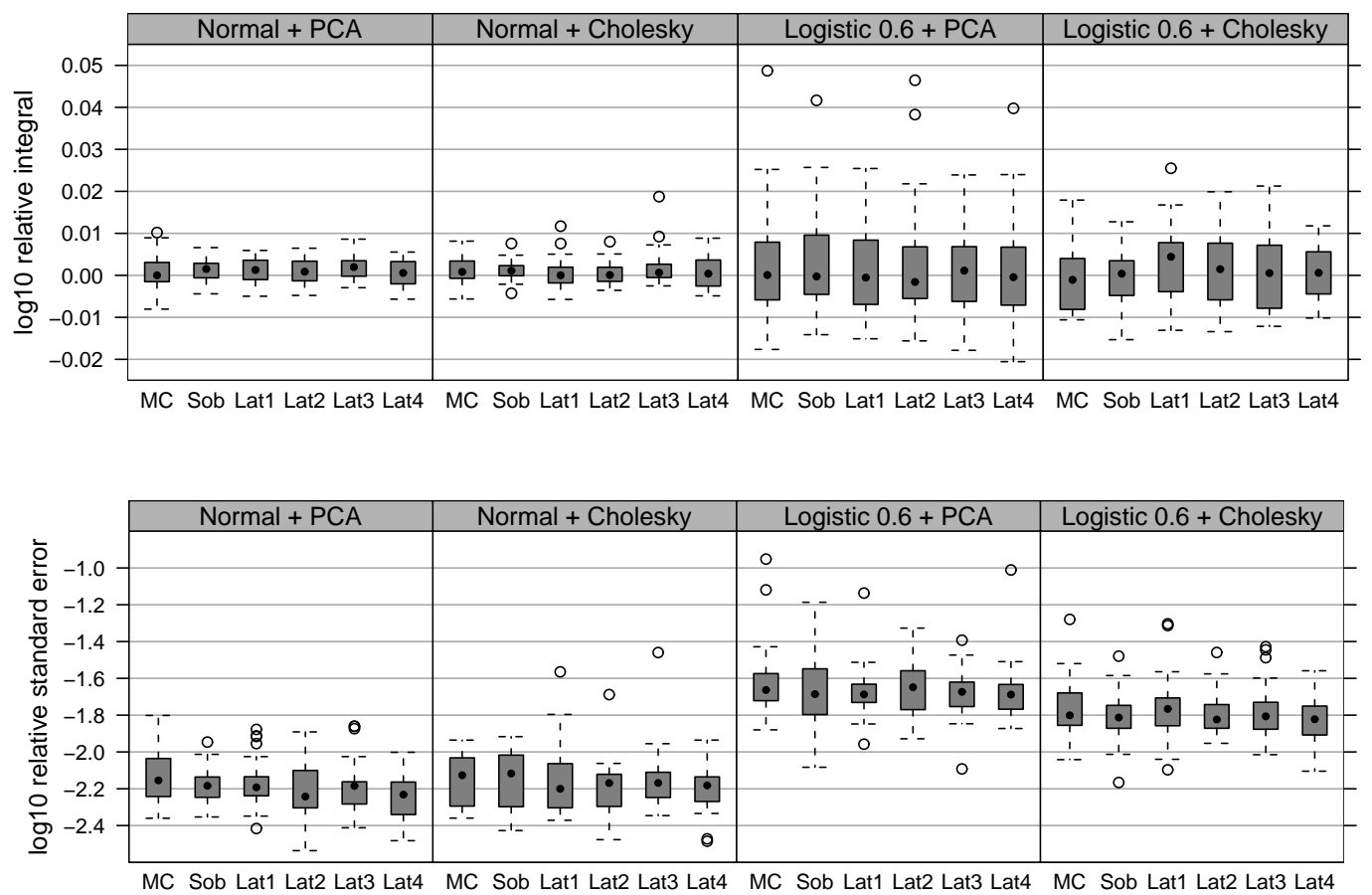

The results for $d=200$ is summarised in Figure 8. At $d=200$, QMC performs only slightly better than $\mathrm{MC}$ under the same transformation, and the best transformation for both MC and QMC appears to be "Normal + PCA". The QMC results under "Logistic $0.6+\mathrm{PCA}^{\prime}$ and "Logistic $0.6+$ Cholesky" are in fact worse than the MC results under "Normal + PCA" or "Normal + Cholesky". The improvement of QMC over MC, both under "Normal + PCA", is a factor of merely 1.13.

To provide a readable scale, three outliers have been cropped out of the MC plot for "Normal + Cholesky": their $\log _{10}$ relative integral values are roughly $3.37,2.91$, and 1.88 . These gross outliers correspond to integration points being extremely close to the boundaries of the unit cube where the transformed integrand is unbounded. Since the "Normal + Cholesky" transformation can lead to more problem boundaries (see the Appendix), it is understandable that we see more outliers in this case.

Recall that when $d=25$ in Example 1 the best QMC transformation is "Logistic $0.6+$ Cholesky". As $d$ increases we observe that the best QMC transformation becomes "Normal + PCA". One possible explanation might be that in higher dimensions the region where the transformed integrand under "Normal + PCA" is unbounded is relatively tiny, while in most parts of the unit cube the integrand is almost constant. Thus the chance of getting an integration point in this tiny region is fairly slim. (We increased $N$ to about one million and the best QMC transformation is still "Normal + PCA". However, with this many points, we ended up with many MC points close to the problem region under 
"Normal + Cholesky" and this resulted in massive outliers.)

\section{Optimisation Using Quasi-Newton}

The maximum likelihood approach seeks the parameter estimates $(\boldsymbol{\beta}, \boldsymbol{\Sigma})$ that maximise the $\log$-likelihood $\ell(\boldsymbol{\beta}, \boldsymbol{\Sigma})$. Efficient numerical methods (see e.g. Fletcher, 1987; Nocedal $\&$ Wright, 1999) require at least the gradient and an approximation of the Hessian of $\ell(\boldsymbol{\beta}, \boldsymbol{\Sigma})$. A quasi-Newton method uses difference in the gradients from successive iterations to build up an approximation to the Hessian, in combination with a line search to gain improvements in the objective. Here we provide only a brief outline of the optimisation process for Example 1 and highlight some important issues.

Let $\boldsymbol{\theta}=(\phi, \sigma, \beta)^{\top}$ denote the vector of parameters in Example 1, let $\ell(\boldsymbol{\theta})$ denote the log-likelihood associated with $\boldsymbol{\theta}$, and let $L(\boldsymbol{\theta})=-\ell(\boldsymbol{\theta}) / \ell\left(\boldsymbol{\theta}^{(0)}\right)$ denote the objective function (which we aim to "minimise"), where $\boldsymbol{\theta}^{(0)}$ is our starting point, for example, the values based on the Laplace approximation. (Note that for both Examples 1 and 2, the objective function values and its derivatives are expensive to obtain, but the number of parameters is relatively small.)

We need the gradient vector $\nabla L(\boldsymbol{\theta})=\left(\frac{\partial L}{\partial \phi}, \frac{\partial L}{\partial \sigma}, \frac{\partial L}{\partial \beta}\right)^{\top}$ as a function of $\boldsymbol{\theta}$. If $I \equiv I(\boldsymbol{\theta})$ denotes the integral $\int_{\mathbb{R}^{d}} \exp \{F(\mathbf{w})\} \mathrm{d} \mathbf{w}$ as a function of $\boldsymbol{\theta}$, then we need to evaluate

$$
\begin{aligned}
\frac{\partial I}{\partial \phi} & =-\frac{\phi}{1-\phi^{2}} I-\frac{1}{2} \int_{\mathbb{R}^{d}} \exp \{F(\mathbf{w})\}\left(\mathbf{w}^{\top} \boldsymbol{\Sigma}_{\phi}^{-1} \mathbf{w}\right) \mathrm{d} \mathbf{w}, \\
\frac{\partial I}{\partial \sigma} & =-\frac{d}{\sigma} I-\frac{1}{2} \int_{\mathbb{R}^{d}} \exp \{F(\mathbf{w})\}\left(\mathbf{w}^{\top} \boldsymbol{\Sigma}_{\sigma}^{-1} \mathbf{w}\right) \mathrm{d} \mathbf{w}, \\
\frac{\partial I}{\partial \beta} & =\left(\mathbf{1}^{\top} \mathbf{y}\right) I-e^{\beta} \int_{\mathbb{R}^{d}} \exp \{F(\mathbf{w})\}\left(\mathbf{1}^{\top} e^{\mathbf{w}}\right) \mathrm{d} \mathbf{w},
\end{aligned}
$$

where

$$
\boldsymbol{\Sigma}_{\phi}^{-1}:=\frac{\partial \boldsymbol{\Sigma}^{-1}}{\partial \phi}=\frac{1}{\sigma^{2}}\left[\begin{array}{cccccc}
0 & -1 & 0 & \cdots & 0 & 0 \\
-1 & 2 \phi & -1 & \cdots & 0 & 0 \\
0 & -1 & 2 \phi & \cdots & 0 & 0 \\
\vdots & \vdots & \vdots & \ddots & \vdots & \vdots \\
0 & 0 & 0 & \cdots & 2 \phi & -1 \\
0 & 0 & 0 & \cdots & -1 & 0
\end{array}\right] \text { and } \boldsymbol{\Sigma}_{\sigma}^{-1}:=\frac{\partial \boldsymbol{\Sigma}^{-1}}{\partial \sigma}=-\frac{2}{\sigma} \boldsymbol{\Sigma}^{-1}
$$

Thus to obtain $L(\boldsymbol{\theta})$ and its gradient $\nabla L(\boldsymbol{\theta})$ for each $\boldsymbol{\theta}$, we need to evaluate the integral $I(\boldsymbol{\theta})$ plus three additional integrals.

Since the integrals will be estimated by the averages of transformed integrand values, to ensure that the estimate of the gradient vector is continuous and consistent, we need to use the same integration rule and the same transformation method for all four integrals. In other words, rather than finding the correct centre and scaling for each integrand separately, we use the centre and scaling obtained for the integrand in $I(\boldsymbol{\theta})$ in all four integral calculations. Fortunately, the integrands in these three additional integrals are just the integrand in $I(\boldsymbol{\theta})$ multiplied by simple terms like $\mathbf{w}^{\top} \boldsymbol{\Sigma}_{\phi}^{-1} \mathbf{w}, \mathbf{w}^{\top} \boldsymbol{\Sigma}_{\sigma}^{-1} \mathbf{w}$, and $\mathbf{1}^{\top} e^{\mathbf{w}}$, which do not have much impact on the feature of the integrand. 
Starting with a parameter vector $\boldsymbol{\theta}^{(0)}$ and an approximate Hessian $\mathbf{B}^{(0)}$ at $\boldsymbol{\theta}^{(0)}$ (initially $\mathbf{B}^{(0)}=\mathbf{I}$ is often used), in step $k$ we need to solve

$$
\mathbf{B}^{(k)} \mathbf{d}^{(k)}=-\nabla L\left(\boldsymbol{\theta}^{(k)}\right)
$$

for the search direction $\mathbf{d}^{(k)}$. Then the next iterate is given by

$$
\boldsymbol{\theta}^{(k+1)}:=\boldsymbol{\theta}^{(k)}+\alpha^{(k)} \mathbf{d}^{(k)},
$$

where $\alpha^{(k)}$ is the minimiser of $l_{k}(\alpha)=L\left(\boldsymbol{\theta}^{(k)}+\alpha \mathbf{d}^{(k)}\right)$ obtained by an approximate line search based on modeling $l_{k}(\alpha)$ by a quadratic or cubic polynomial in $\alpha$ and then determining its stationary points by making use of $l_{k}^{\prime}(\alpha)=\mathbf{d}^{(k)^{\top}} \nabla L\left(\boldsymbol{\theta}^{(k)}\right)$. The approximate Hessian can be updated using, for example, the BFGS update

$$
\mathbf{B}^{(k+1)}:=\mathbf{B}^{(k)}+\frac{\mathbf{t}^{(k)} \mathbf{t}^{(k)^{\top}}}{\mathbf{s}^{(k)^{\top}} \mathbf{t}^{(k)}}-\frac{\mathbf{v}^{(k)} \mathbf{v}^{(k)}}{\mathbf{s}^{(k)^{\top}} \mathbf{v}^{(k)}},
$$

where

$$
\begin{aligned}
\mathbf{s}^{(k)} & :=\boldsymbol{\theta}^{(k+1)}-\boldsymbol{\theta}^{(k)}=\alpha^{(k)} \mathbf{d}^{(k)} \\
\mathbf{t}^{(k)} & :=\nabla L\left(\boldsymbol{\theta}^{(k+1)}\right)-\nabla L\left(\boldsymbol{\theta}^{(k)}\right) \\
\mathbf{v}^{(k)} & :=\mathbf{B}^{(k)} \mathbf{s}^{(k)}=-\alpha^{(k)} \nabla L\left(\boldsymbol{\theta}^{(k)}\right) .
\end{aligned}
$$

The approximate line search conditions ensure that $\mathbf{s}^{(k)^{\top}} \mathbf{t}^{(k)}>0$ so that if $\mathbf{B}^{(0)}$ is positive definite then all subsequent $\mathbf{B}^{(k)}$ are positive definite. The quasi-Newton approximation satisfies the quasi-Newton relation $\mathbf{B}^{(k+1)} \mathbf{s}^{(k)}=\mathbf{t}^{(k)}$, thus $\mathbf{B}^{(k+1)}$ includes information from the difference in gradients $\mathbf{t}^{(k)}$ over the step $\mathbf{s}^{(k)}$.

We stress once again that it is important to use the same transformation and point set for both the objective value and its gradient, so that the derivatives are exact for the approximated objective. If this is not done, then both the line search and the gradient difference used to update the Hessian approximation can be affected. However, changing the parameter vector $\boldsymbol{\theta}^{(k)}$ changes the correct centre and scaling for the integrands. Experiments from the previous section show that the centring and rescaling play a crucial role in the transformation process. It is therefore essential that we carry out only a small number of iterations with a fixed centre and scaling to update the parameter vector. Then we restart the process with the correct centre and scaling to the updated parameter vector. The Hessian approximation obtained at the end of each each round can be used as an initial approximation in the next round. Since the objective function arising from a log-likelihood model is often close to a convex quadratic at the optimal parameters, no more than $p+1$ steps of a $p$-parameter model should be done before updating the centre and scaling. Thus for the objective $L(\boldsymbol{\theta})$ in Example 1, we should carry out no more than 4 steps.

Another issue is when to stop the optimisation iteration. Convergence of an optimisation algorithm is typically based on the change in the objective function $L\left(\boldsymbol{\theta}^{(k+1)}\right)-$ $L\left(\boldsymbol{\theta}^{(k)}\right)$ and the norm $\left\|\nabla L\left(\boldsymbol{\theta}^{(k)}\right)\right\|_{\infty}$ becoming small. However, it makes no sense trying 
to push these below the estimated standard error in the MC or QMC integral calculations. Thus as the optimisation iterates converge, a strategy for increasing the number of integration points is required.

Preliminary calculations indicate that a reasonable approximation of the maximum likelihood parameters can be obtained with just a few optimisation rounds, each with a small number of iteration steps. The Laplace approximation provides a very good starting point for the optimisation process. The best combination of optimisation steps with fixed centre and scaling and the strategy for increasing the number of integration points still requires further study.

\section{Summary}

We carried out numerical experiments with the log-likelihood integrals from a Poisson state-space time-series regression and a Poisson state-space linear mixed model. In both cases, the transformation which brings the integrand into the unit cube plays a crucial role because it determines the features of the transformed integrands. For small dimensions such as $d=25$, QMC dramatically outperforms MC. However, as $d$ increases to 200, the superiority of QMC over MC is diminished.

\section{Appendix}

\subsection{Error Estimation Using Random Shifts}

We now discuss how to use randomly-shifted QMC methods to provide error estimations.

Let $\Delta$ denote a real vector in the unit cube, which we will refer to as the shift. The $\Delta$-shift of a QMC method with points $\xi_{1}, \ldots, \xi_{N}$ is a QMC method with points

$$
\operatorname{frac}\left(\xi_{i}+\Delta\right), \quad i=1, \ldots, N
$$

where $\operatorname{frac}(\mathbf{x})$ is the vector obtained by replacing each component of $\mathbf{x}$ by its fractional part. It can be easily proved that the family of shifted QMC methods is an unbiased estimator of the true integral $I f$.

In practice, for a chosen QMC method $Q_{N}$, we generate a number of independent random shifts $\Delta_{1}, \Delta_{2}, \ldots, \Delta_{r}$ and form the approximations $Q_{N}^{(1)}, Q_{N}^{(2)}, \ldots, Q_{N}^{(r)}$, where $Q_{N}^{(k)}$ is the approximation of the integral If using a $\Delta_{k}$-shift of $Q_{N}$. Then we take the average

$$
\bar{Q}_{N}:=\frac{1}{r}\left(Q_{N}^{(1)}+Q_{N}^{(2)}+\cdots+Q_{N}^{(r)}\right)
$$

as our final approximation to $I f$. An unbiased estimate for the standard error of $\bar{Q}_{N}$ is given by

$$
\sqrt{\frac{1}{r} \frac{1}{r-1} \sum_{k=1}^{r}\left(Q_{N}^{(k)}-\bar{Q}_{N}\right)^{2}} .
$$




\subsection{Estimation of the Effective Dimensions}

Here we outline the techniques described in Wang \& Fang (2003) and Liu \& Owen (2006) for estimating the effective dimensions. Using the identity

$$
T_{\mathfrak{u}}:=\sum_{v \subseteq \mathfrak{u}} \sigma^{2}\left(f_{v}\right)=\int_{[0,1]^{2 d-|\mathfrak{u}|}} f(\mathbf{z}) f\left(\mathbf{z}_{\mathfrak{u}}, \mathbf{w}_{-\mathfrak{u}}\right) \mathrm{d} \mathbf{z} \mathrm{d} \mathbf{w}_{-\mathfrak{u}}-\left(\int_{[0,1]^{d}} f(\mathbf{z}) \mathrm{d} \mathbf{z}\right)^{2},
$$

we can estimate the truncation dimension $d_{T}$ by approximating $T_{\{1\}}, T_{\{1,2\}}, T_{\{1,2,3\}}, \ldots$ until the value reaches $99 \%$ of the total variance $\sigma^{2}(f)$. Here $\left(\mathbf{z}_{\mathfrak{u}}, \mathbf{w}_{-\mathfrak{u}}\right)$ denotes the vector whose $j$ th component is $z_{j}$ if $j \in \mathfrak{u}$ and $w_{j}$ if $j \notin \mathfrak{u}$. If $\left\{\boldsymbol{\xi}_{1}, \ldots, \boldsymbol{\xi}_{N}\right\}$ and $\left\{\boldsymbol{\zeta}_{1}, \ldots, \boldsymbol{\zeta}_{N}\right\}$ are the points of two $d$-dimensional MC or QMC rules, then $T_{\mathfrak{u}}$ can be approximated by

$$
T_{\mathfrak{u}} \approx \frac{1}{N} \sum_{i=1}^{N} f\left(\boldsymbol{\xi}_{i}\right) f\left(\boldsymbol{\xi}_{i, \mathfrak{u}}, \boldsymbol{\zeta}_{i,-\mathfrak{u}}\right)-(I f)^{2},
$$

where $I f$, as well as $\sigma^{2}(f)$, can be estimated using $\left\{\boldsymbol{\xi}_{1}, \ldots, \boldsymbol{\xi}_{N}\right\}$. Thus the cost of estimating $d_{T}$ is the evaluation of at most $d+2$ integrals. The superposition dimension $d_{S}$ can be estimated by making use of $T_{\mathfrak{u}}$ recursively. However, unless the integrals involved were computed very accurately, the propagation of the errors makes it impossible to get a realistic answer for $d_{S}$. The mean dimension $d_{M}$ can be computed using the simple identity

$$
d_{M}=\frac{1}{\sigma^{2}(f)} \sum_{\ell=1}^{d}\left(\frac{1}{2} \int_{[0,1]^{d+1}}\left(f(\mathbf{z})-f\left(\mathbf{w}_{\{\ell\}}, \mathbf{z}_{-\{\ell\}}\right)\right)^{2} \mathrm{~d} \mathbf{z} \mathrm{d} \mathbf{w}_{\{\ell\}}\right),
$$

which requires the evaluation of $d+2$ integrals.

\subsection{Component-by-component Construction of Generating Vectors}

Recall that the lattice points generated by $\boldsymbol{\eta}$ are given by

$$
\xi_{i}=\operatorname{frac}\left(\frac{i \boldsymbol{\eta}}{N}\right), \quad i=1, \ldots, N
$$

For shifted rank-1 lattice rules with random shifts, we consider a "shift-averaged" worstcase error expression for our lattice rule generated by $\boldsymbol{\eta}=\left(\eta_{1}, \ldots, \eta_{d}\right)$, which simplifies to (taking squares)

$$
e_{N}^{2}\left(\eta_{1}, \ldots, \eta_{d}\right)=\frac{1}{N} \sum_{i=1}^{N} \sum_{\emptyset \neq \mathfrak{u} \subseteq\{1, \ldots, d\}}\left(\gamma_{\mathfrak{u}} \prod_{j \in \mathfrak{u}} B_{2}\left(\operatorname{frac}\left(\frac{i \eta_{j}}{N}\right)\right)\right),
$$

where $B_{2}(z)=z^{2}-z+1 / 6$ is the Bernoulli polynomial of degree 2 . We shall not go into the detail of the derivation of this expression. For a given $N$ and a set of weights $\gamma_{\mathfrak{u}}$, the components of a generating vector $\boldsymbol{\eta}$ will be constructed one at a time as follows:

1. Set $\eta_{1}=1$. 
2. For each $d=2,3, \ldots$, with $\eta_{1}, \ldots, \eta_{d-1}$ fixed, choose $\eta_{d}$ from the set $\{1 \leq \eta \leq N-1$ : $\operatorname{gcd}(\eta, N)=1\}$ to minimise $e_{N}\left(\eta_{1}, \ldots, \eta_{d-1}, \eta_{d}\right)$.

This construction is now known in the QMC community as the component-by-component construction (e.g. Sloan et al., 2002). It has been proved that this construction leads to lattice rules that achieve an error of order $\mathcal{O}\left(N^{-1+\delta}\right), \delta>0$, which is the optimal rate of convergence possible in our Sobolev space $H$ (Kuo, 2003). The implied constant in the big- $\mathcal{O}$ notation is independent of $d$ provided that our weights $\gamma_{\mathfrak{u}}$ satisfy a certain condition.

Under the product weight setting, the total computational cost of this algorithm is only $\mathcal{O}(N \log (N) d)$ operations by making use of fast Fourier transforms (Nuyens \& Cools, 2006). Similar reductions in computational cost are also possible for the order-dependent weight setting.

\subsection{Closer Examination of Transformation 1 with $b(x)=e^{x}$}

Clearly the dominating terms in the exponent of $f_{1}$ are the exponential and the quadratic terms, which can be written together as

$$
-\sum_{j=1}^{d} v_{j} \exp \left(\left(\mathbf{W A}^{*} \Psi^{-1}(\mathbf{z})\right)_{j}\right)+\frac{1}{2} \sum_{j=1}^{d} v_{j}\left(\mathbf{W A}^{*} \Psi^{-1}(\mathbf{z})\right)_{j}^{2},
$$

where $\mathbf{v}:=\exp \left(\mathbf{X} \boldsymbol{\beta}+\mathbf{W w}^{*}\right)$, and $\left(\mathbf{W A} \mathbf{A}^{*} \Psi^{-1}(\mathbf{z})\right)_{j}$ denotes the $j$ th component of $\mathbf{W A} \mathbf{A}^{*} \Psi^{-1}(\mathbf{z})$.

Consider the situation where one component of $\mathbf{z}$, say $z_{k}$, goes to either 0 or 1 , while all other components are away from the boundaries. Then the $k$ th component of $\Psi^{-1}(\mathbf{z})$ goes to $\pm \infty$ and all other components are finite. In this case, the vector $\mathbf{W A}^{*} \Psi^{-1}(\mathbf{z})$ contains either 0 or $\pm \infty$ depending on the signs of the entries in the $k$ th column of WA* For example,

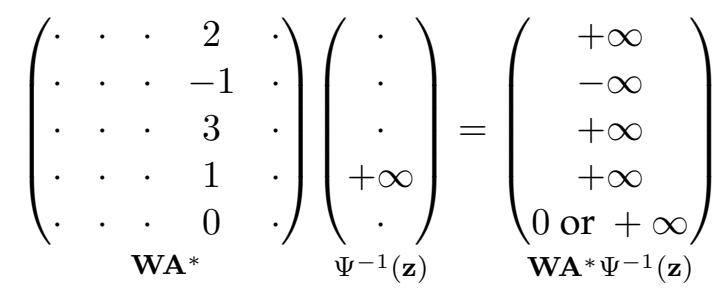

It is obvious that the expression (12) goes to $-\infty$ if any component of $\mathbf{W A}^{*} \Psi^{-1}(\mathbf{z})$ goes to $+\infty$; otherwise the expression goes to $+\infty$. Thus $f_{1}(\mathbf{z}) \rightarrow 0$ if any component of $\mathbf{W A}^{*} \Psi^{-1}(\mathbf{z})$ goes to $+\infty$; otherwise $f_{1}(\mathbf{z}) \rightarrow \infty$. To be more precise, the unboundedness occurs exactly when

- the $k$ th column of $\mathbf{W A}^{*}$ contains no negative entry and $z_{k} \rightarrow 0$;

- the $k$ th column of $\mathbf{W A}^{*}$ contains no positive entry and $z_{k} \rightarrow 1$.

Now we focus on Example 1 in which $\mathbf{W}=\mathbf{I}$. First we take $\mathbf{A}^{*}$ to be the PCA factor of $\Sigma^{*}$. It turns out that only the first column of $\mathbf{A}^{*}$ contains all positive entries; all other columns contain entries with mixed signs. Thus $f_{1}(\mathbf{z})$ is only unbounded when $z_{1} \rightarrow 0$. On the other hand, the Cholesky factor of $\Sigma^{*}$ has no negative entry in any column. In this case $f_{1}(\mathbf{z})$ is unbounded whenever $z_{k} \rightarrow 0$ for any $k$. 


\subsection{Details About the Numerical Experiments in Section 5}

\section{Precisions, random number generator, inverse normal, Sobol' points}

The matrix and vector arithmetics are carried out using LAPACK (Anderson et al., 1999) in double precision. However, the accumulation of all integrals and the associated standard error estimates are done in long double precision.

We use a modified version of the random number generator ran2 by L'Ecuyer from Numerical Recipes in C (Press et al., 1995); we changed it to long double precision and we removed the guard for numbers getting close to 1 . The inverse cumulative normal distribution function is computed using the online algorithm by Acklam (2006). It is accurate to full machine precision.

The parameters for the Sobol' points are obtained from Joe and Kuo (2003), see also Bratley and Fox (1988).

\section{Truncation and mean dimensions}

Following Section 8.2, we estimate the truncation and mean dimensions for Examples 1 and 2 using both Monte Carlo points and Sobol' points. We use 500000 points for $d=$ 25, 50, 100 and 1000000 points for $d=150,200$. Each calculation is done with a single shift, and we repeat the calculation ten times. The number we report is the average of 20 results consisting of ten $\mathrm{MC}$ results and ten Sobol' results.

\section{Two-dimensional projections}

Figures 4 and 5 contain some two-dimensional projections corresponding to various transformed integrands for Example 1 with $d=25$.

The graphs (a.1), (a.2), and (a.3) correspond to the transformed integrand obtained by using "Normal + PCA". In particular, graph (a.1) is the project of $z_{1}$ and $z_{5}$ through the centre of the unit cube $(0.5, \ldots, 0.5)$, graph (a.2) is the projection of $z_{3}$ and $z_{25}$ through the point $(0.3, \ldots, 0.3)$, and graph (a.3) is the projection of $z_{7}$ and $z_{11}$ through the point $(0.8, \ldots, 0.8)$. The graphs (b.1), (b.2), and (b.3) are obtained with the same parameters, except that they correspond to the transformed integrand obtained by using "Logistic 0.6 + Cholesky".

Graph (c) corresponds to the transformed integrand obtained without doing centring or rescaling. It uses the normal distribution and the PCA factor of the original covariance matrix $\Sigma$. The graph is the projection of $z_{1}$ and $z_{5}$ through the centre of the unit cube.

Graph (d) corresponds to the transformed integrand obtained with centring but without rescaling. It uses the logistic distribution with $\lambda=1$ and the PCA factor of the original covariance matrix $\Sigma$. The graph is the projection of $z_{1}$ and $z_{5}$ through the centre of the unit cube.

\section{Choices of lattice rules}

In our numerical experiments we used lattice rules constructed from four different choices of weight settings, including two "finite-order and order-dependent weights", one "equal 
Table 1: Lattice rules constructed based on four choices of weights.

\begin{tabular}{|c|c|l|}
\hline Lat1 & $\begin{array}{l}\text { ORDER TWO } \\
\Gamma_{1}=\Gamma_{2}=1, \\
\Gamma_{\ell}=0 \forall \ell \geq 3\end{array}$ & $\begin{array}{l}\text { All second order interactions are equally } \\
\text { important and there is no higher order } \\
\text { interaction. }\end{array}$ \\
\hline Lat2 & $\begin{array}{c}\text { ORDER THREE } 0.5 \\
\Gamma_{1}=\Gamma_{2}=1, \Gamma_{3}=0.5, \\
\Gamma_{\ell}=0 \forall \ell \geq 4\end{array}$ & $\begin{array}{l}\text { The third order interactions are only half as } \\
\text { important as the second order interactions and } \\
\text { there is no higher order interaction. }\end{array}$ \\
\hline Lat3 & $\begin{array}{c}\text { EQUAL PRODUCT } \\
\gamma_{\{j\}}=0.5, \\
\text { or } \Gamma_{\ell}=0.5^{\ell} \forall \ell \geq 1\end{array}$ & $\begin{array}{l}\text { All variables are equally important, and the } \\
\text { higher order interactions become less and less } \\
\text { important by a factor of } 0.5 .\end{array}$ \\
& $\begin{array}{c}\text { DECAYING PRODUCT } \\
\gamma_{\{j\}}=1 / j\end{array}$ & $\begin{array}{l}\text { The importance of successive variables decays } \\
\text { like } 1 / j \text {, and the weight associated with any } \\
\text { group of variables is simply the product of the } \\
\text { weights for those variables in this group. }\end{array}$ \\
\hline
\end{tabular}

product weights", and one "decaying product weights", see Table 1.

\section{Acknowledgments}

The support of the Australian Research Council under its Centres of Excellence program is gratefully acknowledged. The first author was supported by a University of New South Wales Vice-Chancellor's Postdoctoral Research Fellowship. The authors are thankful for comments from Richard Davis, Yanan Fan, Berwin Turlach, and Henryk Woźniakowski.

\section{References}

Acklam, P.J. (2006). An algorithm for computing the inverse normal cumulative distribution function. http://home. online.no/ pjacklam/notes/invnorm/.

Al-Eid, E. and Pan, J. (2005). Estimation in generalized linear mixed models using SNTO approximation. In: Proceedings of the 20th International Workshop on Statistical Modelling, Sydney, Australia, Francis, A.R., Matawie, K.M., Oshlack, A., and Smyth, G.K. (eds.), pp. 77-84.

Anderson, E., Bai, Z., Bischof, C., Blackford, S., Demmel, J., Dongarra, J., Du Croz, J., Greenbaum, A., Hammarling, S., McKenney, A. and Sorensen, D. (1999). LAPACK Users' Guide, Third Edition. Philadelphia: SIAM.

Bernat, D., Dunsmuir, W.T.M. and Wagenaar, A.C. (2004). Effects of lowering the BAC limit to .08 on fatal traffic crashes in 19 states. Accident Analysis and Prevention, 36, 1089-1097. 
Bratley, P. and Fox, B.L. (1988). Algorithm 659: Implementing Sobol's quasirandom sequence generator. ACM Transactions on Mathematical Software, 14, 88-100.

Breslow, N.E. and Clayton, D.G. (1993). Approximate inference in generalized linear mixed models. Journal of the American Statistical Association, 88, 9-25.

BUGS Project (2006). BUGS: Bayesian Inference Using Gibbs Sampling. http: //www.mrc-bsu.cam.ac.uk/bugs.

Caflisch, R.E., Morokoff, W. and Owen, A. (1997). Valuation of mortgage backed securities using Brownian bridges to reduce effective dimension. Journal of Computational Finance, 1, 27-46.

Clayton, D. (1996). Generalized linear mixed models. In: Markov Chain Monte Carlo in Practice, Gilks, W.R., Richardson, S. and Spiegelhalter, D.J. (eds.), pp. 275-301, London: Chapman \& Hall.

Cools, R., Kuo, F.Y. and Nuyens, D. (2006). Constructing embedded lattice rules for multivariate integration. SIAM Journal on Scientific Computing, to appear.

Crainiceanu, C., Ruppert, D. and Wand, M.P. (2005). Bayesian analysis for penalised spline regression using WinBUGS. Journal of Statistical Software, Volume 14, Issue 14 .

Davis, R.A., Dunsmuir, W.T.M. and Wang, Y. (1999). Modelling Time Series of Count Data. In Asymptotics, Nonparametrics and Time Series S. Ghosh (eds.), pp. 63-114, New York: Marcel-Dekker.

Davis, R.A., Dunsmuir, W.T.M. and Wang, Y. (2000). On autocorrelation in a Poisson regression model. Biometrika, 87, 491-505.

Davis, R.A. and Yodriguez-Yam, G. (2005). Estimation for state-space models based on a likelihood approximation. Statistica Sinica, 15, 81-406.

Dick, J., Pillichshammer, F. and Waterhouse, B.J. (2006). The construction of good extensible rank-1 lattices. Submitted.

Diggle, P., Liang, K.-L. and Zeger, S. (1995). Analysis of Longitudinal Data. Oxford: Oxford University Press.

Diggle, P., Heagerty, P., Liang, K.-L. and Zeger, S. (2002). Analysis of Longitudinal Data, Second Edition. Oxford: Oxford University Press.

Fang, K.-T. and Wang, Y. (1994). Number-Theoretic Methods in Statistics. London: Chapman \& Hall. 
Fletcher, R. (1987). Practical Methods of Optimisation, Second Edition. Chichester and New York: John Wiley.

Gurrin, L.C., Scurrah, K.J. and Hazelton, M.L. (2005). Tutorial in biostatistics: spline smoothing with linear mixed models. Statistics in Medicine, 24, 3361-3381.

Hickernell, F.J. and Hong, H.S. (2002). Quasi-Monte Carlo methods and their randomisations. In: Applied Probability, Chan, R., Kwok, Y.-K., Yao, D. and Zhang, Q. (eds.), AMS/IP Studies in Advanced Mathematics, Vol. 26, pp. 59-77, Providence: American Mathematical Society.

Hickernell, F.J., Lemieux, C. and Owen, A.B. (2005). Control Variates for Quasi-Monte Carlo. Statistical Science, 20, 1-31.

Joe, S. and Kuo, F.Y. (2003). Remark on Algorithm 659: Implementing Sobol's quasirandom sequence generator. ACM Transactions on Mathematical Software, 29, 49-57.

Kuo, F.Y. (2003). Component-by-component constructions achieve the optimal rate of convergence for multivariate integration in weighted Korobov and Sobolev spaces. Journal of Complexity, 19, 301-320.

Kuo, F.Y., Wasilkowski, G.W. and Waterhouse, B.J. (2006). Randomly-shifted lattice rules for unbounded integrands. Journal of Complexity, 22, 630-651.

Kuo, F.Y. and Sloan, I.H. (2005). Lifting the curse of dimensionality. Notices of the American Mathematical Society, 52, 1320-1328.

L'Ecuyer, P. and Lemieux, C. (2002). Recent advances in randomized quasi-Monte Carlo methods. In: Modeling Uncertainty: An Examination of Stochastic Theory, Methods, and Applications, Dror, M., L'Ecuyer, P., and Szidarovszki, F. (eds.), pp. 419-474, Kluwer, Dordrecht.

Liu, R. and Owen, A. (2006). Estimating mean dimensionality of analysis of variance decompositions. Journal of the American Statistical Association, 101, 712-721.

McCulloch, C.E. and Searle, S.R. (2000). Generalized, Linear, and Mixed Models. New York: John Wiley and Sons.

Niederreiter, N. (1992). Random Number Generation and Quasi-Monte Carlo Methods. Philadelphia: SIAM.

Niederreiter, N. (2005). Construction of $(t, m, s)$-nets and $(t, s)$-sequences. Finite Fields and Their Applications, 11, 578-600.

Nocedal, J. and Wright, S.J. (1999). Numerical Optimization. Springer. 
Nuyens, D. and Cools, R. (2006). Fast algorithms for component-by-component construction of rank-1 lattice rules in shift-invariant reproducing kernel Hilbert spaces. Mathematics of Computation, 75, 903-920.

Pan, J.-X. and Thompson, R. (1998). Quasi-Monte Carlo EM algorithm for estimation in generalized linear mixed models. In: Proceedings in Computational Statistics, Payne, R. and Green, P. (eds.), pp. 419-424, Physical-Verlag.

Pan, J. and Thompson, R. (2004). Quasi-Monte Carlo estimation in generalized linear mixed models. In: Proceedings of the 19th International Workshop on Statistical Modelling, Biggeri, A., Dreassi E., Lagazio C. Marchi M. (eds.), pp. 239-243, FLorence: Firenze University Press.

Pan, J. and Thompson, R. (2006). Quasi-Monte Carlo approximation for estimation in generalized linear mixed models. Submitted.

Paskov, S.H. and Traub, J.F. (1995). Faster valuation of financial derivatives. Journal of Portfolio Management, 22, 113-120.

Press, W.H., Teukolsky, S.A., Vetterling, W.T. and Flannery, B.P. (1995). Numerical Recipes in $C$, Second Edition. Cambridge University Press.

Ruppert, D., Wand, M.P. and Carroll, R.J. (2003). Semiparametric Regression. New York: Cambridge University Press.

SAS Institute, Inc. (2006). http: / /www. sas . com.

Skrondal, A. and Rabe-Hesketh, S. (2004). Generalized Latent Variable Modelling: Multilevel, Longitudinal and Structural Equation Models. Boca Raton, Florida: Chapman and Hall.

Sloan, I.H. and Joe, S. (1994). Lattice Methods for Multiple Integration. Oxford: Oxford University Press.

Sloan, I.H., Kuo, F.Y. and Joe, S. (2002). Constructing randomly shifted lattice rules in weighted Sobolev spaces. SIAM Journal on Numerical Analysis, 40, 1650-1665.

Sloan, I.H., Wang, X. and Woźniakowski, H. (2004). Finite-order weights imply tractability of multivariate integration. Journal of Complexity, 20, 46-74.

Sloan, I.H. and Woźniakowski, H. (1998). When are quasi-Monte Carlo algorithms efficient for high dimensional integrals? Journal of Complexity, 14, 1-33.

Wand, M. P. (2003). Smoothing and mixed models. Computational Statistics, 18, 223-249.

Wang, X. and Fang, K.T. (2003). Effective dimensions and quasi-Monte Carlo integration. 
Journal of Complexity, 19, 101-124.

Wolfinger, R. and O'Connell, M. (1993). Generalized linear mixed models: a pseudolikelihood approach. Journal of Statistical Computation and Simulation, 48, 233-243.

Zhao, Y., Staudenmayer, J., Coull, B.A. and Wand, M.P. (2006). General design Bayesian generalized linear mixed models. Statistical Science, 21, 35-51. 